\title{
An improved-Rhie-Chow interpolation scheme for the smoothed-interface immersed boundary method
}

DOI:

$10.1002 / f l d .4240$

\section{Document Version}

Accepted author manuscript

Link to publication record in Manchester Research Explorer

\section{Citation for published version (APA):}

Yi, W., Corbett, D., \& Yuan, X-F. (2016). An improved-Rhie-Chow interpolation scheme for the smoothed-interface immersed boundary method. International Journal for Numerical Methods in Fluids. https://doi.org/10.1002/fld.4240

\section{Published in:}

International Journal for Numerical Methods in Fluids

\section{Citing this paper}

Please note that where the full-text provided on Manchester Research Explorer is the Author Accepted Manuscript or Proof version this may differ from the final Published version. If citing, it is advised that you check and use the publisher's definitive version.

\section{General rights}

Copyright and moral rights for the publications made accessible in the Research Explorer are retained by the authors and/or other copyright owners and it is a condition of accessing publications that users recognise and abide by the legal requirements associated with these rights.

\section{Takedown policy}

If you believe that this document breaches copyright please refer to the University of Manchester's Takedown Procedures [http://man.ac.uk/04Y6Bo] or contact uml.scholarlycommunications@manchester.ac.uk providing relevant details, so we can investigate your claim.

\section{OPEN ACCESS}




\begin{abstract}
(1) 0
e

An improved-Rhie-Chow interpolation scheme for the

\author{
smoothed-interface immersed boundary method \\ Wei $\mathrm{Yi}^{* 1,2}$, Daniel Corbett ${ }^{1}$, Xue-Feng Yuan $^{1,3}$ \\ ${ }^{1}$ Manchester Institute of Biotechnology, The School of Chemical Engineering and Analytical Science, \\ The University of Manchester, Manchester, United Kingdom \\ ${ }^{2}$ State key Laboratory of High Performance Computing, The School of Computer, \\ National University of Defense Technology, Changsha, P. R. China \\ Sun Yat-Sen University, Guangzhou, P. R. China
}

${ }^{3}$ National Supercomputer Centre in Guangzhou, Research Institute on Application of High Performance Computing, S National Supercomputer

SUMMARY

Rhie-Chow interpolation is a commonly used method in CFD calculations on a co-located mesh in order to suppress non-physical pressure oscillations arising from chequer-board effects. A fully parallelised smoothed-interface immersed boundary method on a co-located grid is described in this paper. We discuss the necessity of modifications proposed by Choi [1] to the original Rhie-Chow interpolation in order to deal with a locally refined mesh. Numerical simulation with the modified scheme of Choi shows that numerical dissipation due to Rhie-Chow interpolation introduces significant errors at the immersed boundary. To address this issue we develop an improved-Rhie-Chow interpolation scheme which is shown to increase the accuracy in resolving the flow near the immersed boundary. We compare our improved scheme with the modified scheme of Choi by parallel simulations of benchmark flows i/ flow past a stationary cylinder, ii/ flow past an oscillating cylinder and iii/ flow past a stationary elliptical cylinder, where Reynolds numbers are tested in the range $10-200$. Our improved scheme is significantly more accurate and compares favourably with a staggered grid algorithm. We also develop a scheme to compute the boundary force for the directforcing immersed boundary method efficiently. Copyright (c) 2016 John Wiley \& Sons, Ltd.
\end{abstract}

Received

KEY WORDS: Rhie-Chow interpolation; immersed boundary method; OpenFOAM; finite volume method

${ }^{*}$ Correspondence to: State key Laboratory of High Performance Computing, The School of Computer, National University of Defense Technology, Changsha, P. R. China, Tel: +86-13564101298 Email:yiwei@ nudt.edu.cn

This article has been accepted for publication and undergone full peer review but has not been through the copyediting, typesetting, pagination and proofreading process, which may lead to differences between this version and the Version of Record. Please cite this article as doi: $10.1002 / f l d .4240$

This article is protected by copyright. All rights reserved. 


\section{INTRODUCTION}

For the simulation of fluid flow with moving objects of complex geometry, a conventional method is to dynamically update the mesh according to the new positions of the moving objects, which is referred as remeshing. The dynamic mesh method has the advantage of a well-controlled accuracy in enforcing the boundary condition on the surfaces of moving objects at an expensive computational cost. An alternative method without such expensive remeshing procedure is the immersed boundary method, where moving objects are tracked by a moving Lagrangian grid while the fluid flow is solved on a stationary Cartesian grid. It is especially favorable for simulating fluid flow with multiple moving objects and in parallel computing.

The immersed boundary method can be categorized as smoothed-interface immersed boundary method, where the boundary is smeared out, or the sharp-interface immersed boundary method. The former is usually first-order accurate in space for flow near a sharp boundary while the latter can be second-order or higher. The original immersed boundary method belongs to the smoothed category. It was proposed by Peskin [2] for simulating blood flow around heart valves. Exchanging information between the Lagrangian and Eulerian grids is through a smoothed delta function so the flow field is smeared out in the vicinity of the immersed boundary. Smoothed delta functions [3] should follow fundamental principles, such as momentum conservation, torque conservation etc. An immersed boundary is accomplished by imposing a boundary force, which is introduced into the momentum equation in a way similar to a body force. The boundary force on Lagrangian points can be obtained by solving a constitutive model (Hookean spring [2]), a feedback force model [4] or using the direct forcing method [5]. The direct forcing method has recently gained more popularity [6, 7, 8, 9] because it requires no additional parameters and is suitable for problems with rigid immersed objects. However, due to the use of a smoothed delta function, the flow at a sharp interface is difficult to resolve. The sharp-interface immersed boundary method is favored when boundary layer effects are significant to the entire flow domain, especially for turbulent flow. This method usually involves a local reconstruction of velocity and pressure at the grid near the immersed boundary. It has been widely used in simulating flow with relatively high Reynolds number [10, 11, 12, 13, 14]. Second-order accuracy has been achieved at the fluid-solid interface. However there are also problems which restrict its application. The local reconstruction performed by linear, bi-linear or quadratic interpolation [10, 11] cannot guarantee mass conservation. Additionally, with a moving boundary on top of a Cartesian grid, physical variables in a Cartesian cell can suddenly transit between the solid phase and the fluid phase, leading to serious spurious oscillations [15, 16] in the pressure field. These spurious oscillations can be attenuated by a field extension strategy with some ghost cells inside the immersed object [12] and the mass conservation can be improved by using a more delicate mesh to resolve the geometry of the boundary with cut-cell techniques [17, 18]. In comparison, the oscillation is much less significant with a smoothed-interface immersed boundary method for the simulation of moving boundary problems [6, 19].

Reviewing previous implementations of immersed boundary methods, we find that staggered grids has been chosen in most cases. The co-located grid method is widely-known to be vulnerable to the chequer-board effect where nonphysical pressure oscillations appear. Udaykumar et al. [17] proposed a co-located sharp-interface immersed boundary method with an improved Rhie-Chow interpolation. Mittal et al. [13] also implemented a sharp-interface immersed boundary method on

This article is protected by copyright. All rights reserved. 
a co-located grid. The Mittal group calculated the surface flux by directly taking into account the pressure gradient on cell-faces. Their method can be regarded as a simplified version of the original Rhie-Chow interpolation [20] considering only the diagonal coefficients from the temporal term but without the implicit viscous term in the momentum equation. Therefore nonphysical pressure oscillation could still appear when the viscous implicit terms dominate. The investigation of a smoothed-interface immersed boundary method on a co-located mesh was not found in literatures. With the original Rhie-Chow interpolation, Choi found that the steady state solution was dependent on the time step, Yu et al. [21] suggested that the chequer-board effect would still appear if a small time step is used. A modification was proposed to address this issue by considering a separate treatment of the temporal term in the interpolation [1, 21]. A general summary of the Rhie-Chow interpolation can be found in [22].

In this paper we will discuss several issues related to the implementation of a co-located smoothed-interface immersed boundary solver. Major contributions of this paper include:

(i) We develop a numerical method for a smoothed-interface immersed boundary method with an improved-Rhie-Chow interpolation scheme. To our knowledge, this is the first of such scheme which uses a co-located grid.

(ii) The accuracy of enforcing the no-slip boundary condition at the immersed boundary is typically increased by either using more forcing iterations or decreasing the time step (or both) [8, 23]. We develop a more cost-effective scheme by adopting an initial estimate for the boundary force based on its value in the previous time step. We validate our new scheme against benchmark flows.

(iii) We have analysed the error introduced by the original Rhie-Chow interpolation and the modified Rhie-Chow interpolation [1]. The latter is necessary when dealing with locally refined meshes. Various types of Rhie-Chow interpolation is found to produce significant numerical dissipation near an immersed boundary where the pressure is discontinuous [24, 25]. We develop an improved-Rhie-Chow algorithm which significantly reduces the dissipation close to the immersed boundary. We compare our improved-Rhie-Chow interpolation with Choil's modified version in two benchmark simulations and demonstrate our method is able to resolve flows near the immersed boundary at higher accuracy.

(iv) Our algorithms are developed in OpenFOAM, an open-source finite volume method (FVM) solver written in $\mathrm{C}++$. OpenFOAM provides many data-structures particularly suited for CFD calculations (vectors, 2nd rank tensors...) and also makes parallelisation of algorithms simple and efficient.

\section{NUMERICAL METHOD}

\subsection{Momentum equation discretization}

The motion of an incompressible fluid flow is governed by the momentum equation and the continuity equation, written as

$$
\partial_{t} \underline{u}+\underline{u} \cdot \nabla \underline{u}=-\nabla p+\frac{\eta}{\rho_{f}} \nabla^{2} \underline{u}+\underline{f}
$$

This article is protected by copyright. All rights reserved. 


$$
\nabla \cdot \underline{u}=0
$$

where $\eta$ is the dynamic viscosity of the fluid, $\rho_{f}$ is the density of the fluid, $p$ is the kinematic pressure, $\partial_{t}$ indicates a partial derivative with respect to time, $f$ is the boundary force (per unit mass) in the vicinity of the immersed boundary. The force density $f$ is described in section 2.3 .

The convection term is discretized with the second-order Adam-Bashforth scheme. The diffusion term and the boundary force term are discretized with the second-order Crank-Nicholson scheme. Thus the overall accuracy of the numerical scheme is second-order in time.

$$
\frac{\underline{u}^{n+1}-\underline{u}^{n}}{\Delta t}=-\frac{3}{2} C\left[\underline{u}^{n}\right]+\frac{1}{2} C\left[\underline{u}^{n-1}\right]+\frac{\eta}{2 \rho_{f}}\left(\nabla^{2} \underline{u}^{n}+\nabla^{2} \underline{u}^{n+1}\right)-\nabla p^{n+\frac{1}{2}}+\frac{1}{2}\left(\underline{f}^{n}+\underline{f}^{n+1}\right)
$$

where $C$ is the convection operator and the superscript indicates the time step.

The momentum equation is solved with a co-located finite volume method. The integral of the momentum equation in a control volume involves the computation of several face-centre quantities from cell-centre variables, including face-centre velocity, flux, face-centre gradient and face-centre gradient along the normal direction. The face-centre velocity is obtained by mid-point interpolation from the cell-centre velocity. The face-centre gradient is computed with a second-order central difference scheme. Rhie-Chow interpolation is used for computing the flux in order to avoid the well-known chequer-board effect (see section 2.4). In the presence of a local refined mesh, an over-relaxed non-orthogonal correction [26] is chosen to correct the error in gradient at interfaces between the coarse and the refined mesh grid, where the connection between cell centres are not aligned with the face normal.

The discretized momentum equation can be reformulated as follows:

$$
a_{P} \underline{u}_{P}^{n+1}=H_{P}-(\nabla p)_{P}+\frac{\underline{u}_{P}^{n}}{\Delta t}
$$

where $a_{P}$ is the diagonal coefficient of the linear equations, $\underline{u}_{P}^{n} / \Delta t$ is the explicit part of the time derivative term $\partial_{t} \underline{u}_{P}, H_{P}$ is a combination of implicit off-diagonal terms, other explicit terms and source terms of the momentum equation.

\subsection{Immersed boundary method}

The immersed boundary method uses a separate Lagrangian mesh to track the surface of immersed objects. Figure 1 shows an immersed boundary (circle points) on a uniform Eulerian grid. The boundary condition can be enforced by boundary forces on Lagrangian points. The Lagrangian force is spread to nearby Eulerian grid inside a support cage (e.g. the $4 \times 4$ dashed square in Figure 1 for the gray circular Lagrangian point). The Navier-Stokes equation is solved on the Eulerian grid with the finite volume method. Information exchange between the Eulerian and Lagrangian grid generally consists of two steps: one step is to calculate the velocity of the Lagrangian points by an interpolation from the velocity of the Eulerian points; the other step is to spread the aforementioned force from Lagrangian points to the Eulerian points in their support cages. For clarity, the variables for Lagrangian points are denoted by capital letters, e.g. the boundary force $\underline{F}$ and the Lagrangian velocity $\underline{U}$. The boundary force (per unit mass) on Eulerian points (achieved by spreading) is introduced to the momentum equation as a source term $\underline{f}$, as shown in eqns. 1 and 3 . The size

This article is protected by copyright. All rights reserved. 
of the support cage is determined based on the effective domain of the smoothed delta function, which is a numerical approximation of the Dirac Delta function. In this paper, we consider IB3 [27] and IB4 [2]. More smoothed delta functions as well as the design rule are described in [3]. The interpolation and spreading procedures are shown in eqn. 5 and 6 . The formulation of IB3 and IB4 are shown in eqn. 8 and eqn. 9

$$
\begin{aligned}
\underline{U}(X) & =\int \underline{u}(x) \delta(x-X(s)) d x \\
\underline{f}(x) & =\int \underline{F}(s) \delta(x-X(s)) d s
\end{aligned}
$$

where $\delta$ is the Dirac Delta function, its discretized form in 2D is shown in eqn. 7

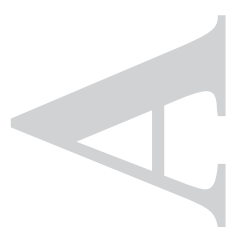

$$
\begin{gathered}
\left.\delta(x, y)=\phi\left(\frac{x}{\Delta x}\right) \phi\left(\frac{y}{\Delta x}\right)\right) / \Delta x^{2} \\
\phi^{I B 3}(r)= \begin{cases}\frac{1}{3}\left(1+\sqrt{-3 r^{2}+1}\right) & |r| \leq 0.5 \\
\frac{1}{6}\left(5-3|r|-\sqrt{\left.-3(1-|r|)^{2}+1\right)}\right. & 0.5 \leq|r| \leq 1.5 \\
0 & |r| \geq 1.5\end{cases} \\
\phi^{I B 4}(r)= \begin{cases}\frac{1}{8}\left(3-2|r|+\sqrt{1+4|r|-4 r^{2}}\right) & 0 \leq|r| \leq 1 \\
\frac{1}{8}\left(5-2|r|-\sqrt{-7+12|r|-4 r^{2}}\right) & 1 \leq|r| \leq 2 \\
0 & 2 \leq|r|\end{cases}
\end{gathered}
$$

The Lagrangian force $\underline{F}$ for a no-slip boundary condition is computed with a prediction of velocity $\underline{U}^{*}$ at Lagrangian points in the absent of an immersed boundary. Several different models for computing the boundary force have been proposed in [2, 4, 5]. The direct forcing method [5] is a simple and efficient method since there is no additional artificial parameters for the simulation. The boundary force can be estimated by $\underline{F}^{(*)}=\frac{\underline{U}_{0}-\underline{U}^{(*)}}{\Delta t}$, where $\underline{U}_{0}$ is the target velocity on the boundary. For stationary no-slip boundary, $\underline{U}_{0}=0 . \underline{U}^{(*)}$ is a prediction of Lagrangian velocity from Eulerian velocity $\underline{u}^{(*)}$ through eqn. 5 . A full derivation for the evaluation of boundary force is shown in [7] and intensively discussed in [23].

\subsection{An iterative forcing scheme}

The velocity boundary condition on the surface of the immersed object is enforced with an iterative forcing scheme. The necessity of iteration loop is the reason that the calculation of the boundary force is roughly based on the diagonal terms of the linear equations, as shown in eqn. 10 . The interpolated velocity at the Lagrangian points does not meet the no-slip boundary condition with one iteration. An iteration loop was suggested in $[8,23]$ to iteratively update the boundary force and velocity explicitly without re-solving the momentum equations. The accuracy of enforcing the noslip boundary condition can be improved by using smaller time steps or more forcing iterations. The iterative forcing scheme we use has two major differences when compared with previous iterative schemes: (a) the initial velocity prediction is obtained with an initial prediction of the boundary force from previous time step, which is assumed to be zero in all previous schemes; (b) the computation 
of boundary force on the Lagrangian points is done according to eqn. 11 based on the diagonal coefficient of the linear equations (eqn. 47). The difference from the treatment of forcing with eqn. 10 and 11 vanishes as the time step reduces. No significant difference is shown when simulation results from the two formulations are compared.

At the beginning of each time iteration, the velocity is predicted by solving the momentum equation with a fully-explicit scheme, shown in eqn. 12 .

$$
\begin{gathered}
\underline{F}^{\prime}=\frac{\underline{U}_{0}-\underline{U}^{*}}{\Delta t} \\
\underline{F}^{\prime}=a_{p}\left(\underline{U}_{0}-\underline{U}^{*}\right)
\end{gathered}
$$

where $a_{p}$ is the diagonal coefficient of the linear equations.

$$
\frac{\underline{u}^{n+1}-\underline{u}^{n}}{\Delta t}=-\frac{3}{2} C\left[\underline{u}^{n}\right]+\frac{1}{2} C\left[\underline{u}^{n-1}\right]+\frac{\eta}{\rho_{f}} \nabla^{2} \underline{u}^{n}-\nabla p^{n}+\underline{f}^{n}
$$

The algorithm for the iterative forcing procedure is summarized in the following six steps: step 0: $n$ Iter $=0$;

step 1: use the force on Lagrangian points from the previous time step with $\underline{F}^{*}=\underline{F}^{n}$, and spread $\underline{F}^{*}$ to Eulerian grid $\underline{f}^{*}$ according to eqn. 6 ,

step 2: solve the momentum equation explicitly with eqn. 12 to get a prediction of the velocity field $\underline{u}^{*}$;

step 3: interpolate the velocity from the Eulerian grid $\underline{u}^{*}$ to the Lagrangian grid $\underline{U}^{*}$ with eqn. 5 .

step 4: evaluate the boundary force correction $\underline{F}^{\prime}$ with eqn. 11 and update the boundary force $\underline{F}^{*}=\underline{F}^{*}+\underline{F}^{\prime}$

step 5: solve the momentum equation implicitly with eqn. 3 ;

step 6: $n$ Iter $=$ nIter +1 , if nIter $<$ forceIter return to step 3 .

It is worth mentioning that although the pressure correction procedure after solving the momentum equation makes changes to the velocity field and therefore affects the accuracy for enforcing the no-slip boundary condition. The changes associated with the pressure correction procedure are insignificant and negligible.

\subsection{Rhie-Chow interpolation}

2.4.1. original scheme Consider the discretization of the momentum equation onto a uniform grid in the form of eqn. 4 for the cell $P$ and its east neighbour $E$ with face $e$ between them,

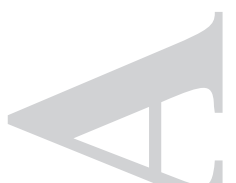

$$
\begin{aligned}
& a_{P} \underline{u}_{P}^{n+1}=H_{P}-(\nabla p)_{P}+\frac{\underline{u}_{P}^{n}}{\Delta t} \\
& a_{E} \underline{u}_{E}^{n+1}=H_{E}-(\nabla p)_{E}+\frac{\underline{u}_{E}^{n}}{\Delta t}
\end{aligned}
$$

The Rhie-Chow interpolation proposed by Rhie and Chow [20] computes the face-centre velocity as follows,

$$
a_{e} \underline{u}_{e}^{n+1}=\left(H_{E}+H_{P}\right) / 2-(\nabla p)_{e}+\frac{\left(\underline{u}_{P}^{n}+\underline{u}_{E}^{n}\right)}{2 \Delta t}
$$

This article is protected by copyright. All rights reserved. 
where $a_{e}=a_{P}=a_{E}$ for a uniform grid and $(\nabla p)_{e}=\left(p_{E}-p_{P}\right) / \Delta x$, notice $(\nabla p)_{e} \neq$ $\frac{1}{2}\left[(\nabla p)_{P}+(\nabla p)_{E}\right]$.

Consider the $1 \mathrm{D}$ case, $\nabla p$ is replaced by $\frac{\partial p}{\partial x}$. We can obtain the following equation from eqns. 13 . 14 and 15 .

$$
\underline{u}_{e}^{n+1}=\frac{1}{2}\left(\underline{u}_{P}^{n+1}+\underline{u}_{E}^{n+1}\right)-\frac{1}{a_{e}}\left[\left(\frac{\partial p}{\partial x}\right)_{e}-\frac{1}{2}\left(\frac{\partial p}{\partial x}\right)_{P}-\frac{1}{2}\left(\frac{\partial p}{\partial x}\right)_{E}\right]
$$

According to the Taylor series expansions, the Rhie-Chow interpolation introduces a third-order derivative term of the pressure in space to the velocity as follows,

$$
\left(\frac{\partial p}{\partial x}\right)_{e}-\frac{1}{2}\left(\frac{\partial p}{\partial x}\right)_{P}-\frac{1}{2}\left(\frac{\partial p}{\partial x}\right)_{E}=-\frac{\Delta x^{2}}{8}\left(\frac{\partial^{3} p}{\partial x^{3}}\right)_{e}+O\left(\Delta x^{4}\right)
$$

When the viscous term in the momentum equation is discretised explicitly, $a_{e}=\rho / \Delta t$. As a result, eqn. 16 becomes,

$$
\underline{u}_{e}^{n+1}=\frac{1}{2}\left(\underline{u}_{P}^{n+1}+\underline{u}_{E}^{n+1}\right)+\frac{\Delta x^{2}}{8} \frac{\Delta t}{\rho}\left(\frac{\partial^{3} p}{\partial x^{3}}\right)_{e}
$$

In our numerical implementation, we calculate the flux based in the absence of the pressure contribution as follows,

$$
a_{e} \underline{u}_{e}^{*}=\left(H_{E}+H_{P}\right) / 2+\frac{\left(\underline{u}_{P}^{n}+\underline{u}_{E}^{n}\right)}{2 \Delta t}
$$

The pressure field can then be solved with the following equation,

$$
\frac{1}{a_{P}} \nabla^{2} p=\nabla \cdot \underline{u}^{*}
$$

The cell-centre velocity field is then updated with the pressure field according to,

$$
\underline{u}^{n+1}=\underline{u}^{*}-\frac{\nabla p}{a_{P}}
$$

It can be proved that solving the pressure with eqns. 19 and 20 is equivalent to the original RhieChow interpolation where a correction of pressure is solved together with eqn. 15

We summarise the pressure correction algorithm in the following, step 0: nIter=0;

step 1: calculate the flux with eqn. 19, note the operator $H$ involving implicit terms from neighbouring cells is also updated;

step 2: solve the pressure with eqn. 20 and update the velocity field $\underline{u}^{n+1}$ with eqn. 21 ,

step 3: nIter $=$ nIter +1 , if $n$ Iter $<$ pressureIter return to step 1 .

2.4.2. modified-Rhie-Chow interpolation scheme A modified-Rhie-Chow scheme was suggested in [1, 21] because the original scheme was found to have some weak points: (a) the steady state solution depends on the time step length [1], (b) chequer-board effects still appear if a small time step is used [21]. A remedy was introduced considering a separate treatment of the temporal term in the interpolation [1, 21] shown below,

This article is protected by copyright. All rights reserved. 


$$
a_{e} \underline{u}_{e}^{n+1}=\left(H_{E}+H_{P}\right) / 2-(\nabla p)_{e}+\frac{\underline{u}_{e}^{n}}{\Delta t}
$$

where $\underline{u}_{e}^{n}$ is the face-centre velocity calculated in previous time step. In practice, the flux at old time step is stored rather than the face-centre velocity for the convenience of implementation.

We again analyse error terms using the 1D case. From eqns. 13, 14 and 25, the modified-RhieChow interpolation can be described by,

$$
\underline{u}_{e}^{n+1}=\frac{1}{2}\left(\underline{u}_{P}^{n+1}+\underline{u}_{E}^{n+1}\right)-\frac{1}{a_{e}}\left[\left(\frac{\partial p}{\partial x}\right)_{e}-\frac{1}{2}\left(\frac{\partial p}{\partial x}\right)_{P}-\frac{1}{2}\left(\frac{\partial p}{\partial x}\right)_{E}\right]+\frac{\underline{u}_{e}^{n}}{\Delta t}-\frac{\left(\underline{u}_{P}^{n}+\underline{u}_{E}^{n}\right)}{2 \Delta t}
$$

Since

$$
\frac{\underline{u}_{e}^{n}}{\Delta t}-\frac{\left(\underline{u}_{P}^{n}+\underline{u}_{E}^{n}\right)}{2 \Delta t}=-\frac{\Delta x^{2}}{8 \Delta t}\left(\frac{\partial^{2} u}{\partial x^{2}}\right)_{e}+O\left(\Delta x^{4} / \Delta t\right)
$$

The modified Rhie-Chow interpolation introduces an additional second-order derivative term of the velocity in space to the velocity as follows,

$$
\underline{u}_{e}^{n+1}=\frac{1}{2}\left(\underline{u}_{P}^{n}+\underline{u}_{E}^{n}\right)+\frac{\Delta x^{2}}{8} \frac{\Delta t}{\rho}\left(\frac{\partial^{3} p}{\partial x^{3}}\right)_{e}+\frac{\Delta x^{2}}{8 \Delta t}\left(\frac{\partial^{2} u}{\partial x^{2}}\right)_{e}+O\left(\Delta x^{4}\right)
$$

$E_{1}$ and $E_{2}$ are used to represent the two dissipation terms,

$$
\begin{gathered}
E_{1}=\frac{\Delta x^{2}}{8} \frac{\Delta t}{\rho}\left(\frac{\partial^{3} p}{\partial x^{3}}\right)_{e} \\
E_{2}=\frac{\Delta x^{2}}{8 \Delta t}\left(\frac{\partial^{2} u}{\partial x^{2}}\right)_{e}
\end{gathered}
$$

With a small time step, $E_{1}$ vanishes while $E_{2}$ becomes significant. $E_{1}$ and $E_{2}$ could introduce large errors when the flow field is discontinuous. In addition, the modified Rhie-Chow interpolation introduces a fourth-order derivative term of pressure and third-order derivative term term of velocity into the continuity equation.

2.4.3. improved-Rhie-Chow interpolation scheme at the immersed boundary In the conventional computing with a body-fitted mesh, Rhie-Chow interpolation is not applied for computing the flux at boundaries because the flux can be achieved based on the boundary conditions. With an immersed boundary method, the internal part of the immersed boundary also participate in solving the Navier-Stokes equations. Due to the enforcing of the no-slip boundary condition with a smoothed boundary force, the pressure field in the vicinity of the immersed surface becomes nonphysical and a discontinuity in the pressure is present even with a staggered grid method (Figure 5 in [7]). Therefore, the discontinuity in pressure should also be maintained for a co-located grid method. However, the numerical dissipation in eqn. 18 involves a third-order derivative of pressure in space. Due to the discontinuity in pressure, the numerical dissipation is large and would have a much more significant impact on the face-centre velocity in comparison to a smooth pressure field. The RhieChow interpolation tends to smooth the pressure profile near the immersed boundary. It is interesting to investigate the effect of Rhie-Chow interpolation in the vicinity of an immersed boundary where a boundary force is present. We developed a numerical solver avoiding Rhie-Chow interpolation at

This article is protected by copyright. All rights reserved. 
the faces where boundary forces are present. More specifically, the cell-centre boundary force on the Eulerian grid $f$ is first interpolated from cell-centres to face-centres. The velocity on faces where the boundary force is not zero is replaced by a simple linear interpolation of the velocity. The new method is termed 'improved-Rhie-Chow interpolation'. As we will show in the results section, our simple remedy can improve accuracy significantly. The new formulation to calculate $\underline{u}^{*}$ is given by,

$$
\left(a_{P}\right)_{e} \underline{u}_{e}^{*}= \begin{cases}\left(H_{E}+H_{P}\right) / 2+\frac{\underline{u}_{P}^{n}+\underline{u}_{E}^{n}}{2 \Delta t}+(\nabla p)_{e}-\frac{1}{2}\left[(\nabla p)_{E}+(\nabla p)_{P}\right] & \left|f_{e}\right|>0 \\ \left(H_{E}+H_{P}\right) / 2+\frac{u_{e}^{n}}{\Delta t} & \left|f_{e}\right|=0\end{cases}
$$

\subsection{Calculation of lift and drag force}

For the simulation of moving objects, the total force and torque on immersed objects are computed. The kinematics of moving objects can be computed by solving the Newton-Euler equations. The total force is an integral of the stress $\underline{\underline{\tau}}$ over the surface. For a smoothed-interface immersed boundary method, it can be evaluated by total change of momentum exerted on the particle by the fluid with eqn. 29[6].

$$
\oint \underline{\underline{\tau}} \cdot d \underline{A}=-\int_{V} \underline{f} d V+\rho_{f} \frac{d}{d t} \int_{V} \underline{u} d V
$$

Similarly, the torque can be calculated with eqn. 30

$$
I_{p} \frac{d \underline{\omega_{c}}}{d t}=-\int_{\partial V} \underline{r} \times \underline{f} d V+\rho_{f} \frac{d}{d t} \int_{\partial V} \underline{r} \times \underline{u} d V
$$

where $I_{p}$ is the momentum of inertia, $I_{p}=\frac{1}{2} m R^{2}$ for a $2 \mathrm{D}$ cylinder, $I_{p}=\frac{2}{5} m R^{2}$ for a sphere.

The detailed derivation of eqn. 29 and 30 can be found in [6]. By assuming rigid motion inside an immersed object, the second term on the right hand side of eqn. 29 is simplified to $V_{p} \frac{d u_{c}}{d t}$ and the second term on the right hand side of eqn. 30 is simplified to $\rho_{f} \int_{V} \underline{r} \times \frac{d \underline{u}}{d t} d V=\frac{\rho_{f} I_{p}}{\rho_{p}} \frac{d \omega_{c}}{d t}$. Kempe and Frhlich [23] suggested it was necessary to evaluate the second term in both eqn. 29 and 30 through an integral by considering internal flow of the immersed object in order to achieve better convergence when the density ratio between the immersed object and the fluid is smaller than unity. They used a second order accuracy Heaviside step function to compute the volume fraction of solid (eqn. 31).

$$
\frac{d}{d t} \int \underline{u} d V=\sum_{i j} \Delta x^{2} \alpha_{i j} \frac{d u_{i j}}{d t}
$$

where $\alpha_{i j}$ is calculated with the Heaviside step function (eqn. 32]. [28]

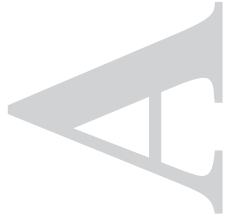

$$
\alpha_{i j}=\frac{\sum_{n=1}^{8}-\phi_{n} H\left(-\phi_{n}\right)}{\sum_{n=1}^{8}\left|\phi_{n}\right|}
$$

where $H()$ is the Heaviside function, $\phi_{n}$ is the signed distance level set function of vertex $n$ (eqn. 33). 


$$
\phi_{n}=\left|{\underline{x_{i j}}}^{n}-\underline{x_{c}}\right|-R
$$

where $x_{i j}^{n}$ is the position vector of the $n^{\text {th }}$ vertex of the grid cell, and $\underline{x_{c}}$ is the position vector of the mass centre, $R$ is the radius.

\subsection{Boundary conditions}

2.6.1. Dirichlet boundary condition When a Dirichlet boundary condition is applied on a boundary, evaluation of surface gradient ( $\mathrm{snGrad}$ ) along the normal direction is discretized by a onesided scheme (OpenFOAM 2.3.1), as is shown in eqn. 34. snGrad is required for finite-volume discretization of the viscous term in the momentum equation.

$$
u^{\prime}=\frac{u_{e}-u_{P}}{\Delta x / 2}
$$

This scheme is first-order accurate for the evaluation of the gradient.

$$
\begin{gathered}
u_{e}=u_{P}+\frac{\Delta x}{2} u_{P}^{\prime}+\frac{\Delta x^{2}}{4} \frac{1}{2} u_{P}^{\prime \prime}+\frac{\Delta x^{3}}{8} \frac{1}{6} u_{P}^{\prime \prime \prime}+O\left(\Delta x^{4}\right) \\
u_{W}=u_{P}-\Delta x u_{P}^{\prime}+\frac{1}{2} \Delta x^{2} u_{P}^{\prime \prime}-\frac{1}{6} \Delta x^{3} u_{P}^{\prime \prime \prime}+O\left(\Delta x^{4}\right) \\
\frac{2\left(u_{e}-u_{P}\right) / \Delta x-\left(u_{P}-u_{W}\right) / \Delta x}{\Delta x}=\frac{3}{4} u_{P}^{\prime \prime}-\frac{1}{8} \Delta x u_{P}^{\prime \prime \prime}+O\left(\Delta x^{2}\right) \\
\frac{\frac{8}{3} u_{e}-4 u_{P}+\frac{4}{3} u_{W}}{\Delta x^{2}}=u_{P}^{\prime \prime}-\frac{1}{6} \Delta x u_{P}^{\prime \prime \prime}+O\left(\Delta x^{2}\right) \\
u_{e}^{\prime}=\frac{\frac{8}{3} u_{e}-3 u_{P}+\frac{1}{3} u_{W}}{\Delta x}+O\left(\Delta x^{2}\right)
\end{gathered}
$$

The Taylor expansion shows the error for Laplacian term in original scheme for handling Dirichlet boundary is $O(1)$ (eqn. 37). However, the scheme can be easily improved by a correction coefficient (eqn. 38). The corresponding equation for evaluation of $s n G r a d$ at the Dirichlet boundary is shown in eqn. 39 with a second-order accuracy in space.

2.6.2. Neumann boundary condition A Neumann boundary condition specifies the gradient along the normal direction. The evaluation of variables on face centres is necessary for the finite-volume discretization of the convection term in the momentum equation. In OpenFOAM 2.3.1, it is also implemented by a one-sided scheme (eqn. 40,.

$$
u_{e} \approx u_{P}+\frac{\Delta x}{2} u_{e}^{\prime}
$$

This article is protected by copyright. All rights reserved. 


$$
u_{e}=u_{P}+\frac{\Delta x}{2} u_{e}^{\prime}-\frac{\Delta x^{2}}{8} u_{e}^{\prime \prime}+O\left(\Delta x^{3}\right)
$$

This scheme is both second-order accurate for the evaluation of the variable on face-centres and the gradient on cell-centres (eqn. 42).

$$
\begin{aligned}
& {\left[35-\frac{1}{2} \times[36-41] / \Delta x\right.} \\
& \quad \frac{u_{P}+\frac{\Delta x}{2} u_{e}^{\prime}-\frac{1}{2}\left(u_{P}+u_{W}\right)}{\Delta x}=u_{P}^{\prime}-\frac{\Delta x}{8}\left(u_{P}^{\prime \prime}-u_{e}^{\prime \prime}\right)+O\left(\Delta x^{2}\right)=u_{P}^{\prime}+O\left(\Delta x^{2}\right)
\end{aligned}
$$

There are higher-order schemes for Dirichlet and Neumann boundary conditions achieved by using large stencil for surface interpolation. However, they are not applicable to OpenFOAM because OpenFOAM uses a sparse matrix to store the linear equation with a data structure that can only store coefficients of nearest neighbours. The order of accuracy at the boundaries does not affect the general purpose of this paper, namely the study of Rhie-Chow intepolation scheme for a smoothed-interface immersed boundary method.

\subsection{Parallel implementation and OpenFOAM}

Our implementation of the immersed boundary method is based on an open source platform OpenFOAM with MPI for inter-processor communication. Each Lagrangian point can be mapped to a single cell in the Eulerian grid. The decomposition of the Lagrangian grid is based on the decomposition of the Eulerian grid. The processor which owns the Eulerian cell also owns Lagrangian points in that specific Eulerian cell. In our test, the finest mesh has 859900 cells, which are distributed to a maximum number of 96 cores for parallel simulations. The velocity equations are solved by a preconditioned bi-conjugate gradient solver. The pressure equations are solved by a preconditioned conjugate gradient solver. Details about the implementation of the finite volume method and the conjugate gradient solver for the linear systems can be found in the user guide [29].

One of the crucial steps for parallel simulation is to quickly locate grid cells in the support cage for a Lagrangian point. The immersed boundary method requires all Lagrangian points to be positioned on the most refined grids. So the searching scheme only need to consider the most refined cells. A quick-search algorithm is designed to determine the processor $I D$ and cell $I D$ for any point in the mesh. During the preprocessing, a global mapping list of (procID, localCellID, globalID) is generated. procID is the processor ID where the cell is distributed to after mesh decomposition. localCellID is the cell ID on the processor. globalID is computed according to eqn. 43 .

$$
\text { globalI } D=\left\lfloor\frac{x-x_{\min }}{\Delta x_{\min }}\right\rfloor \times 2^{s}+\left\lfloor\frac{y-y_{\min }}{\Delta x_{\min }}\right\rfloor
$$

where $x_{\min }$ and $y_{\min }$ are the minimal $x$-coordinate and $y$-coordinate in the full computation domain, $2^{s}$ is the mask for the $x$-direction $\left(y_{\max }<2^{s}\right), \Delta x_{\min }$ is the minimal grid spacing. Each processor stores part of global mapping list as a local mapping list for all local cells and a few layers of remote cells near processor boundaries. An efficient data structure of Hash table or self-balancing binary search tree is used to store the mapping list. The key of the binary search tree is the globalID. For any Lagrangian point, the globalID can be computed with eqn. 43 . Then the time complexity

This article is protected by copyright. All rights reserved. 
for locating a Lagrangian point is $O(\log N)$, where $N$ is length of local mapping list. There is no inter-processor communication involved at this step.

Parallel communication is involved in spreading and interpolating procedures, updating positions of Lagrangian points for moving boundary, as well as computing the total force and torque on the immersed object. The last is trivial with simple reduce and gather operations. For other procedures, the exchange of information between processor is based on a request-and-reply pattern, where the request from one processor to the other is sent in blocks for the purpose of efficiency. The information of a Lagrangian point is stored on the processor where it locates so the locality is greatly explored and inter-processor communication for interpolating and spreading procedures are only necessary when a Lagrangian point is close to a processor boundary. Our scheme is able to support any mesh decomposition scheme and also works for multi-layer Cartesian mesh (Figure 2). We have verified that simulation results are independent of the mesh decomposition scheme and the number of processors used for computing.

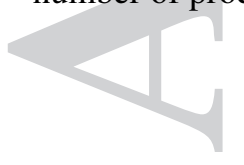

\section{RESULT}

\subsection{Flow solver without an immersed boundary}

The flow solver is firstly investigated through a two-dimensional channel flow. The simulation is carried out on a rectangular domain of $[-6,8] \times[-1,1]$. A no-slip boundary condition is applied at $y= \pm 1$. Periodic boundary conditions are applied at the inlet $x=-6$, and the outlet $x=8$. The implementation of Dirichlet boundary condition is the original OpenFOAM scheme. The flow is driven by a fixed pressure difference between the inlet and outlet. The Reynolds number for this problem is defined by $R e=\rho_{f} \bar{u} H / \eta$, where $\bar{u}$ is the maximum flow rate, $H$ is the height of the channel $(H=2), \rho_{f}$ is the fluid density and $\eta$ the dynamic viscosity. The steady state solution for velocity field can be expressed by $u(y)=\bar{u}\left(1-y^{2}\right)$. The corresponding pressure gradient for driving the flow is $\nabla p_{0}=-8 \eta \bar{u} / H^{2}$. The error of the simulation can be quantitatively evaluated by $L_{\infty}=\max |e(i)|, L_{1}=\sum|e(i)| \Delta V$ and $L_{2}=\sqrt{\sum\left|e^{2}(i)\right| \Delta V}$, where $\Delta V$ is the normalized volume of a mesh cell, $e(i)$ is the error at cell $i$. We measure the error on three meshes $140 \times 20$, $280 \times 40$ and $560 \times 80$. The simulation result shows error for the steady state measured by $L_{1}$, $L_{2}$ and $L_{\infty}$. They are almost the same for different $R e$ with the same grid. The error is shown in table II $L_{\infty}(M 1) / L_{\infty}(M 2)=L_{\infty}(M 2) / L_{\infty}(M 3)=4$. Therefore the solver is second-order accurate in space when there is no immersed boundary. The treatment of the Dirichlet boundary has no significant impact on current measurement of order of accuracy.

\subsection{Effects of forcing iteration, time step and history force}

In section 2.3, we proposed using the boundary force on the Lagrangian points from previous time step as an initial prediction of the boundary force at current time step. To verify the effectiveness of this scheme, we consider the benchmark case of flow past a stationary cylinder. A cylinder with radius $R=0.15$ is placed at $(1,1)$ in a rectangular channel $[0,2] \times[0,2]$. For the velocity, Dirichlet boundary condition $\underline{u}=\left(u_{\infty}, 0\right)$ with $u_{\infty}=1$ is enforced at the side walls $(y=0$ and $y=2)$ and inlet $(x=0)$; Neumann boundary condition $\partial \underline{u} / \partial x=0$ is enforced at the outlet of the flow $(x=2)$.

This article is protected by copyright. All rights reserved. 
For the pressure, Neumann boundary condition $\partial p / \partial \underline{n}=0$ is enforced at the side walls and inlet; Dirichlet boundary condition $p=0$ is set at the outlet. The Reynolds number for this case is defined as $R e=\rho_{f} \bar{u} D / \eta$, where $D$ is the diameter of the cylinder. The improved-Rhie-Chow interpolation scheme is used for calculating the flux on the surface.

Since the expected velocity on Lagrangian points is zero for a no-slip boundary, the velocity at Lagrangian points represents the numerical error. We calculate the velocity at Lagrangian points with the smoothed delta function when steady state is reached. For a specific mesh, it is wellunderstood that this error can be attenuated by increasing the number of forcing iterations or by decreasing the time step [23]. A uniform grid with grid spacing of $1 / 50$ is used. The Lagrangian points are evenly distributed on the surface of the cylinder with $\Delta s \approx \Delta x$, where $\Delta s$ is the arc length between two neighbour Lagrangian points, $\Delta x$ is the grid spacing. Figure 3 shows the error of enforcing the no-slip boundary condition. For the study of forcing iteration effect, the time step is fixed as $\Delta t=0.004$, with a Courant number at around 0.4; for the study of time step effect, the number of forcing iterations is fixed at 4 . The number of pressure correction iterations is fixed to 2 in all simulations in this paper. When the initial prediction of the boundary force is inherited from forces on Lagrangian points at the previous time step, the error is $10^{-7}$ even with 2 forcing iterations and $\Delta t=0.004$. Therefore, we think that the prediction from an inherited force can achieve much higher accuracy at low computational expense. Figure 4 shows the overall profile of $x$-component velocity, $U_{x}$ along the centreline when inherited forces are used. Figure 5(a) and 5 b) shows a magnified view of the $\mathrm{x}$-component of velocity near the front of the cylinder without inherited forces. This figure shows the raw cell-centred data sampled according to the nearest-cell rule. As the number of forcing iterations increases or the time step decreases, the velocity profile along the centre-line approaches the velocity profile predicted with inherited forces. The velocity at the cells close to the Lagrangian point appears to be negative. The error at the cells close to the immersed boundary can be further reduced by grid refinement.

\subsection{Effect of temporal term in the Rhie-Chow interpolation}

The original Rhie-Chow interpolation is able to substantially suppress pressure oscillations due to the chequer-board effect. As pointed out by Choi [1], for time-dependent simulations, the original Rhie-Chow interpolation results in a steady-state solution that is dependent on the timestep. Additionally, the chequer-board effect still appears if the time step is relative small, i.e. when the Courant number is small. The original Rhie-Chow interpolation is likely to cause problems in simulations with a gradient mesh or a locally refined mesh because the maximum time step is limited by the minimal grid spacing, and local Courant number at the less refined region could be very small. Local mesh refinement is widely applied in simulation with immersed boundary method due to the inherently lower order of accuracy for resolving flow near the boundary. Therefore, the temporal term (the second term on the r.h.s of eqn. 25) suggested by Choi [1] becomes crucial for a co-located immersed boundary method. An effective solution is to handle the temporal term separately in the interpolation (see section 2.4.2). We demonstrate the importance of this temporal term through a benchmark case running on a local refined mesh.

The benchmark case of flow past a stationary cylinder is again considered to verify the importance of the temporal correction term in computing face fluxes. The geometry and boundary conditions are the same as in section 3.2 but in a larger computational domain $[0,16] \times[0,16]$. The wall effect

This article is protected by copyright. All rights reserved. 
on the flow near the cylinder is weak. The cylinder is initially placed at $[6.85,8]$. A 4-layer mesh is generated as shown in Figure 2 . The minimal grid spacing for this test is $D / 60$. The Reynolds number is 40 . Nonphysical pressure oscillation is observed to be significant in the coarse mesh region. Figure 6 samples the pressure along the centreline in the range between $x=14$ and $x=16$. The oscillation of pressure becomes more severe when a smaller time step is used. The effect of adding a temporal correction term to the original Rhie-Chow interpolation is clearly shown.

\subsection{Mesh convergence study of the immersed boundary solver}

Since there is no analytical solution for the flow, the mesh convergence study uses the simulation result with a very refined mesh $(800 \times 800)$ as the reference solution for calculating errors. The order of convergence is estimated by $s=\ln \left(e^{(2 h)} / e^{(h)}\right) / \ln 2$, where $e^{(h)}$ and $e^{(2 h)}$ are the error associated with meshes of grid spacing $h$ and $2 h$. The Courant number is kept around 0.4 in all simulations. Table $[$ and $[\mathrm{II}]$ list the order of convergence in space evaluated on four meshes of size $50 \times 50,100 \times 100,200 \times 200$ and $400 \times 400$. Our measurement of convergence rate is similar to the simulation carried out in [13, 23]. The reference solution on mesh $800 \times 800$ is interpolated to coarse meshes with a second-order scheme. The difference between the coarse mesh solution and the reference solution is calculated and its magnitude represents the error. $L_{1}$ and $L_{2}$ are indicators of global accuracy while $L_{\infty}$ demonstrates the error at the immersed boundary. The only difference in the solver between the modified- Rhie-Chow interpolation and the improved-RhieChow interpolation is that the latter avoids Rhie-Chow interpolation in the vicinity of the immersed boundary. With the modified-Rhie-Chow interpolation, the order of accuracy is about 1.3 according to $L_{1}$ and $L_{2}$, but only first-order according to $L_{\infty}$; with the improved-Rhie-Chow interpolation, the order of accuracy is close to first-order according to $L_{\infty}, L_{1}$ and $L_{2}$. The difference in the order of convergence between the two solvers reveals that the global error is dominant by the error introduced at the immersed boundary. Figure 7(a) and 7 (b) show the magnitude of difference in $x$-direction velocity with mesh $400 \times 400$ and $800 \times 800$ for $R e=40$. The improved-Rhie-Chow interpolation has a better resolution of the flow at the boundary especially less penetration at the front of the cylinder.

\subsection{Flow past a stationary cylinder}

To further validate the correctness of our code, we investigate the drag and lift force on a cylinder for different Reynolds number. We use a 4-layer mesh with a computation domain of $[0,16] \times[0,16]$, the same as in section 3.3 . Two different meshes with minimal grid spacing of $D / \Delta x=60$ (meshA) and $D / \Delta x=120$ (meshB) are considered; the number of Lagrangian points around the cylinder are 192 and 384 respectively. Table IV compares the drag coefficient for $R e=20$ and $R e=40$. The results show a good consistency with other publications. For larger $R e$, a Karman vortex street is generated at the rear of the cylinder when a small perturbation in the flow is present. The prediction of the drag and lift coefficients with the improved-Rhie-Chow interpolation shows a better accuracy with the same mesh for larger Re even though both methods are likely to converge to a similar solution if a more refined mesh is used. Figure 9 shows the z-direction vorticity contour for $R e=100$ and $R e=200$ from simulation with meshB. Figure 8 shows the streamline for different 
Reynolds numbers. Similar vorticity profiles and streamlines are observed with the modified-RhieChow solver. The corresponding lift and drag coefficient are shown in table $\mathrm{V}$. The drag and lift coefficients are defined as $C_{d}=F_{x} /\left(\rho u_{\infty}^{2} R\right) ; C_{l}=F_{y} /\left(\rho u_{\infty}^{2} R\right)$, where $F_{x}$ and $F_{y}$ are the drag and lift force on the cylinder. The Strouhal number is defined as $S t=f_{s} D / u_{\infty}$, where $f_{s}$ is the shedding frequency of the Karman vortex. Figure 10 compares the velocity and pressure profile at the front and up point of the cylinder for $R e=20$. The velocity from the improved-Rhie-Chow solver is smoother than the modified-Rhie-Chow solver. On the other hand, the pressure from the improvedRhie-Chow solver is steeper than the modified-Rhie-Chow solver. We also compare velocity and pressure profiles at the front point for $R e=100$ (Figure 11). The above results demonstrate superior predictions when using the improved-Rhie-Chow interpolation scheme.

\subsection{Flow past an oscillatory cylinder}

In this part, a benchmark test with a moving boundary is considered. A cylinder in the free stream is moving with a specified periodic oscillation in the direction perpendicular to the cross flow:

$$
y(t)=y_{0}+A \sin \left(2 \pi f_{e} t\right)
$$

where $y(t)$ is the $y$-coordinate of the cylinder centre, $y_{0}$ is the initial $y$-coordinate, $A$ is the amplitude, $f_{e}$ is the oscillation frequency, $A=0.2 D$ and $f_{e}=0.52$. We choose the same Reynolds number $R e=185$ as in [6]. $f_{e}$ is 0.8 times the natural shedding frequency $f_{o}$ at $R e=185$. The Courant number for all simulations is kept constant at around 0.4. Other configurations are the same as the stationary cylinder case in section 3.5 . Table $\mathrm{VI}$ shows the simulation result when a pseudo-steady state is reached. Our improved-Rhie-Chow solver shows similar predictions compared with that from the smoothed-interface immersed boundary method with a staggered grid [19, 36]. MeshA has a minimal grid spacing of $D / 60$, which is comparable to the grid spacing $D / 50$ used by Yang et al. [19]. The resultant mean drag coefficient and root-mean-square ( $r m s)$ lift coefficient agree well. In comparison to our improved-Rhie-Chow solver, the modified-Rhie-Chow solver has an overestimation of drag of $9.5 \%$ and $5 \%$, and an over-estimation of the rms lift coefficient of $45.8 \%$ and $26.8 \%$ for meshA and meshB. Figure 12 shows the periodic evolution of the drag and lift coefficient as a function of the $y$-coordinate with the modified-Rhie-Chow solver. The comparison on two meshes with grid spacing of $D / 60$ and $D / 120$ shows the prediction of the drag and lift coefficients are still relatively mesh-dependent. Figure 13 shows the corresponding results with the improvedRhie-Chow interpolation scheme, the mesh-dependent variation is much smaller. We also carried out the same simulation with a more compact support cage of $3 \times 3$ grid cells. The selection of support cage has a stronger influence on the modified-Rhie-Chow solver. Figure 12 shows a larger error for the prediction of lift and drag with $I B 3$ than that with $I B 4$. It suggests that the dissipation introduced by Rhie-Chow interpolation is increased when the pressure profile is less smooth with $I B 3$. Simulation with $I B 3$ with the improved-Rhie-Chow solver does not show a significant change int the prediction of drag and lift coefficients (Figure 13. The time evolution of $C_{d}$ and $C_{l}$ with $I B 4$ is smoother than those with IB3. Comparison between the modified-Rhie-Chow solver and the improved-Rhie-Chow solver in Figure 12 and Figure 13 show that the improved-Rhie-Chow scheme does not cause stronger oscillations in the drag and lift coefficients. Our improved-RhieChow solver avoids interpolation at surfaces which have a boundary force and we thus conclude 
that smoothing are not necessary. However, smoothing can easily be incorporated into the solver at an interface where the boundary force is discontinuous across the boundary.

\subsection{Flow past a stationary elliptical cylinder}

We then consider the case flow past a stationary elliptical cylinder. The equation of an elliptical cylinder that orients along the flow is given by,

$$
\frac{\left(x-x_{0}\right)^{2}}{a^{2}}+\frac{\left(y-y_{0}\right)^{2}}{b^{2}}=1
$$

where $a$ and $b$ are the major axis and minor axis. The Lagrangian points are uniformly distributed on the circumference of the cylinder with the same length of arc between neighbouring Lagrangian points. The aspect ratio of the eclipse $(a / b)$ is set as 2 . The same meshes for the simulation of flow past a stationary/oscillating cylinder are used, with minimal grid spacings of $a / 30$ and $a / 60$. The maximum Courant number is kept around 0.3 for all simulations. The Reynolds number for the flow is defined with the major axis of the elliptical cylinder, $R e=\rho U_{\infty} a / \eta_{s}$. Flow past a stationary elliptical cylinder with the attack angle of $\alpha=0, \pi / 6, \pi / 3, \pi / 2$ are simulated. Figure 14 illustrates streamlines around the eclipse with different attack angles when $R e=40$. When the eclipse orients along the flow, the flow is stable with a pair of symmetric vortexes at the rear of the cylinder. As the attack angle increases, flow asymmetry develops and vortexes are generated periodically at the rear of the cylinder. Figure 15 and 16 show the time evolution of drag coefficients when $R e=40$ and $R e=100$ with different meshes and different interpolation schemes. when the time step decreases, change in the drag profiles is found to be insignificant. The drag coefficient of the elliptical cylinder is defined as $C_{d}=F_{d} / \rho u_{\infty}^{2} a$. When the attack angle is $\pi / 6$ or $\pi / 3$, the drag profile in a periodic cycle shows an asymmetric pattern. We can see that the results from the improved-Rhie-Chow interpolation exhibit a weaker mesh dependency in the drag coefficients, in comparison with that from the modified-Rhie-Chow interpolation. The detailed drag coefficient and the Strouhal number are described in Table VII and VIII. The Strouhal number is defined as $S t=2 f_{s} a / u_{\infty}$, where $f_{s}$ is the vortex shedding frequency. There is no significant difference for the prediction of the Strouhal number between the two schemes $(\leq \% 1)$. The solver with the improved-Rhie-Chow interpolation is found to give a better prediction for the drag coefficients.

\subsection{Sedimentation of a pair of cylinders}

The benchmark cases in section 3.5, 3.6 and 3.7 consider either a stationary boundary or a moving boundary with pre-determined motions. In this section, sedimentation of a pair of $2 \mathrm{D}$ cylinders is simulated. The translational and angular velocities are solved with the Newton-Euler equations. The trailing cylinder accelerates faster due to the drag reduction in the wake area of the leading cylinder, thus approaches the leading cylinder quickly, resulting in a draft-kissing-tumbling pattern. Uhlmann [6] studied the problem with a direct-forcing immersed boundary method on a staggered grid. Simulations of this case with other configurations can be found in [37, 38, 39]. Our study considers the same parameters as in [6] (table [X]. The computational geometry is shown in Figure 17.

To avoid the particle collision due to insufficient grid resolution and to resolve the flow field at the gap between the particles, an artificial potential force is enforced when the separation between

This article is protected by copyright. All rights reserved. 
the two cylinders falls below a threshold value [40]. The potential force is given by,

$$
\underline{F}_{i, j}^{P}= \begin{cases}0 & d_{i, j}>2 R+\xi_{P} \\ \frac{1}{\epsilon_{P}}\left(\underline{C}_{i}-\underline{C}_{j}\right)\left(2 R+\xi_{P}-d_{i, j}\right) & d_{i, j} \leq 2 R+\xi_{P}\end{cases}
$$

where $\underline{C}_{i}$ and $\underline{C}_{j}$ are the position vector of the cylinder centre, $d_{i, j}$ is the distance between cylinder centres, $\xi_{P}$ is the threshold separation, $\epsilon_{p}$ is stiffness. In this test, $\epsilon_{p}=5 \times 10^{-7}$ and $\xi_{p}=3 \Delta x$.

The simulation considers the same grid spacing and time step length used in Uhlmann [6], $\Delta x=$ $D / 64$ and $\Delta t=0.0001$. The time-evolution of the displacement and the velocity are monitored. The results with the improved-Rhie-Chow interpolation are shown in Figure 18 . The $x$-direction displacement and velocity, agree closely with those predicted by Uhlmann [6], up to the stage of "kissing". In the tumbling stage, the velocity is strongly affected by the parameters set in the collision model, making it hard to have a qualitatively comparison. In addition, the $x$-direction velocity with the modified-Rhie-Chow and the improved-Rhie-Chow interpolations are compared. The predicted $x$-direction velocity of the leading-cylinder shows a considerable difference with the modified-Rhie-Chow and the improved-Rhie-Chow interpolations even during the drafting period in the absent of the artificial collision force. Such difference still exists when using a finer mesh, smaller time steps, or more iterations. The $x$-direction velocity with the improved-Rhie-Chow interpolation is found to agree with that reported in [6] (Figure 18(b)).

\section{CONCLUSION}

In this paper we have presented a smoothed-interface immersed boundary method on a co-located grid. Rhie-Chow interpolation is often used for calculating surface fluxes in order to avoid chequerboard effects. However, discontinuities in the pressure field at the immersed boundary result in a large dissipation from a standard Rhie-Chow interpolation. This dissipation term is the dominate source of error, and becomes more significant as the Reynolds number increases. We verified this quantitatively by simulating flow past a cylinder. The dissipation could be reduced by mesh refinement at an expensive computational cost. The immersed boundary method with Rhie-Chow interpolation is far less accurate than a staggered grid method using the same mesh. We therefore have developed an improved Rhie-Chow interpolation algorithm which uses standard Rhie-Chow interpolation at surfaces with no boundary force, and avoids interpolation at surfaces with a boundary force. Using this improved Rhie-Chow interpolation with a co-located grid we are able to obtain accuracy matching a staggered grid.

Previous direct forcing immersed boundary methods require a large number of force iterations to accurately enforce the no-slip boundary condition at the immersed boundary. In this paper we have used the boundary force from the previous time step as the initial guess for the boundary force at the current time step. Our subsequent simulations confirm that far fewer force iterations are needed to enforce the no-slip boundary condition with this scheme, even if a large time step is used. Finally, our implementation on OpenFOAM provides a readily scalable platform for 3D simulations. In future work we intend to apply this method to 3D simulations of hard sphere suspensions.

This article is protected by copyright. All rights reserved. 


\section{ACKNOWLEDGEMENT}

Wei Yi's PhD studentship is funded by the University of Manchester and the Chinese Scholarship Council. Wei Yi thanks to N8 HPC facility in Leeds and the National supercomputer centre in Guangzhou for providing computing resources. Wei Yi also thanks to the OpenFOAM team in National University of Defense Technology for helpful discussions.

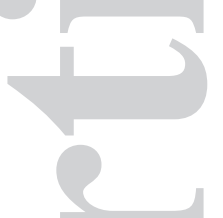

\section{REFERENCES}

[1] Seok Ki Choi. Note on the use of momentum interpolation method for unsteady flows. Numerical Heat Transfer: Part A: Applications, 36(5):545-550, 1999.

[2] Charles S. Peskin. Flow patterns around heart valves: A numerical method. Journal of Computational Physics, 10(2):252-271, 1972.

[3] Charles S. Peskin. The immersed boundary method. Acta Numerica, 11:479-517, 2002.

[4] D. Goldstein, R. Handler, and L. Sirovich. Modeling a no-slip flow boundary with an external force-field. Journal of Computational Physics, 105(2):354-366, 1993. Ku501 Times Cited:269 Cited References Count:34.

[5] J. Mohd-Yusof. Combined immersed-boundary/b-spline methods for simulations of flow in complex geometries. Center for Turbulence Research Annual Research Briefs, pages 317327, 1997.

[6] Markus Uhlmann. An immersed boundary method with direct forcing for the simulation of particulate flows. Journal of Computational Physics, 209(2):448-476, 2005.

[7] A. Pinelli, I. Z. Naqavi, U. Piomelli, and J. Favier. Immersed-boundary methods for general finite-difference and finite-volume navierstokes solvers. Journal of Computational Physics, 229(24):9073-9091, 2010.

[8] W. P. Breugem. A second-order accurate immersed boundary method for fully resolved simulations of particle-laden flows. Journal of Computational Physics, 231(13):4469-4498, 2012. 935JU Times Cited:0 Cited References Count:38.

[9] C. Ji, A. Munjiza, and J. J. R. Williams. A novel iterative direct-forcing immersed boundary method and its finite volume applications. Journal of Computational Physics, 231(4):1797$1821,2012$.

[10] Yu-Heng Tseng and Joel H. Ferziger. A ghost-cell immersed boundary method for flow in complex geometry. Journal of Computational Physics, 192(2):593-623, 2003.

[11] Elias Balaras. Modeling complex boundaries using an external force field on fixed cartesian grids in large-eddy simulations. Computers \& Fluids, 33(3):375-404, 2004.

This article is protected by copyright. All rights reserved. 
[12] Jianming Yang and Elias Balaras. An embedded-boundary formulation for large-eddy simulation of turbulent flows interacting with moving boundaries. Journal of Computational Physics, 215(1):12-40, 2006.

[13] R. Mittal, H. Dong, M. Bozkurttas, F. M. Najjar, A. Vargas, and A. von Loebbecke. A versatile sharp interface immersed boundary method for incompressible flows with complex boundaries. Journal of Computational Physics, 227(10):4825-4852, 2008.

[14] Jianming Yang and Frederick Stern. A simple and efficient direct forcing immersed boundary framework for fluidstructure interactions. Journal of Computational Physics, 231(15):50295061, 2012.

[15] Jongho Lee, Jungwoo Kim, Haecheon Choi, and Kyung-Soo Yang. Sources of spurious force oscillations from an immersed boundary method for moving-body problems. Journal of Computational Physics, 230(7):2677-2695, 2011.

[16] Jung Hee Seo and Rajat Mittal. A sharp-interface immersed boundary method with improved mass conservation and reduced spurious pressure oscillations. Journal of Computational Physics, 230(19):7347-7363, 2011.

[17] H. S. Udaykumar, Heng-Chuan Kan, Wei Shyy, and Roger Tran-Son-Tay. Multiphase dynamics in arbitrary geometries on fixed cartesian grids. Journal of Computational Physics, 137(2):366-405, 1997.

[18] T. Ye, R. Mittal, H. S. Udaykumar, and W. Shyy. An accurate cartesian grid method for viscous incompressible flows with complex immersed boundaries. Journal of Computational Physics, 156(2):209-240, 1999.

[19] Xiaolei Yang, Xing Zhang, Zhilin Li, and Guo-Wei He. A smoothing technique for discrete delta functions with application to immersed boundary method in moving boundary simulations. Journal of Computational Physics, 228(20):7821-7836, 2009.

[20] CM Rhie and WL Chow. Numerical study of the turbulent flow past an airfoil with trailing edge separation. AIAA Journal, 21(11):1525-1532, 1983.

[21] Bo Yu, Yasuo Kawaguchi, Wen-Quan Tao, and Hiroyuki Ozoe. Checkerboard pressure predictions due to the underrelaxation factor and time step size for a nonstaggered grid with momentum interpolation method. Numerical Heat Transfer: Part B: Fundamentals, 41(1): 85-94, 2002.

[22] Sijun Zhang, Xiang Zhao, and Sami Bayyuk. Generalized formulations for the rhiechow interpolation. Journal of Computational Physics, 258(0):880-914, 2014.

[23] Tobias Kempe and Jochen Frhlich. An improved immersed boundary method with direct forcing for the simulation of particle laden flows. Journal of Computational Physics, 231(9): 3663-3684, 2012.

[24] S Venkateswaran and CL Merkle. Evaluation of artificial dissipation models and their relationship to the accuracy of euler and navier-stokes computations. In Sixteenth International Conference on Numerical Methods in Fluid Dynamics, pages 427-432. Springer, 1998.

This article is protected by copyright. All rights reserved. 
[25] F Ham and G Iaccarino. Energy conservation in collocated discretization schemes on unstructured meshes. Annual Research Briefs, 2004:3-14, 2004.

[26] Hrvoje Jasak. Error analysis and estimation for the finite volume method with applications to fluid flows. PhD thesis, 1996.

[27] Alexandre M. Roma, Charles S. Peskin, and Marsha J. Berger. An adaptive version of the immersed boundary method. Journal of Computational Physics, 153(2):509-534, 1999.

[28] Tobias Kempe, Stephan Schwarz, and Jochen Frhlich. Modelling of spheroidal particles in viscous flows. In Proceedings of the Academy Colloquium Immersed Boundary Methods: Current Status and Future Research Directions, 2009.

[29] ESI. Openfoam user guide. http://www.openfoam.com/, 2014.

[30] Mark N. Linnick and Hermann F. Fasel. A high-order immersed interface method for simulating unsteady incompressible flows on irregular domains. Journal of Computational Physics, 204(1):157-192, 2005.

[31] Kunihiko Taira and Tim Colonius. The immersed boundary method: A projection approach. Journal of Computational Physics, 225(2):2118-2137, 2007.

[32] Jung-Il Choi, Roshan C. Oberoi, Jack R. Edwards, and Jacky A. Rosati. An immersed boundary method for complex incompressible flows. Journal of Computational Physics, 224 (2):757-784, 2007.

[33] C. Liu, X. Zheng, and C. H. Sung. Preconditioned multigrid methods for unsteady incompressible flows. Journal of Computational Physics, 139(1):35-57, 1998.

[34] X. Y. Lu and C. Dalton. Calculation of the timing of vortex formation from an oscillating cylinder. Journal of Fluids and Structures, 10(5):527-541, 1996.

[35] E. Guilmineau and P. Queutey. A numerical simulation of vortex shedding from an oscillating circular cylinder. Journal of Fluids and Structures, 16(6):773-794, 2002.

[36] Lennart Schneiders, Daniel Hartmann, Matthias Meinke, and Wolfgang Schrder. An accurate moving boundary formulation in cut-cell methods. Journal of Computational Physics, 235(0): 786-809, 2013.

[37] HowardH Hu, DanielD Joseph, and MarcelJ Crochet. Direct simulation of fluid particle motions. Theoretical and Computational Fluid Dynamics, 3(5):285-306, 1992.

[38] Z Zhang and A Prosperetti. A method for particle simulation. Journal of applied mechanics, 70(1):64-74, 2003.

[39] Saeed Jafari, Ryoichi Yamamoto, and Mohamad Rahnama. Lattice-boltzmann method combined with smoothed-profile method for particulate suspensions. Physical Review E, 83 (2):026702, 2011.

[40] R. Glowinski, T. W. Pan, T. I. Hesla, and D. D. Joseph. A distributed lagrange multiplier/fictitious domain method for particulate flows. International Journal of Multiphase Flow, 25(5):755-794, 1999.

This article is protected by copyright. All rights reserved. 

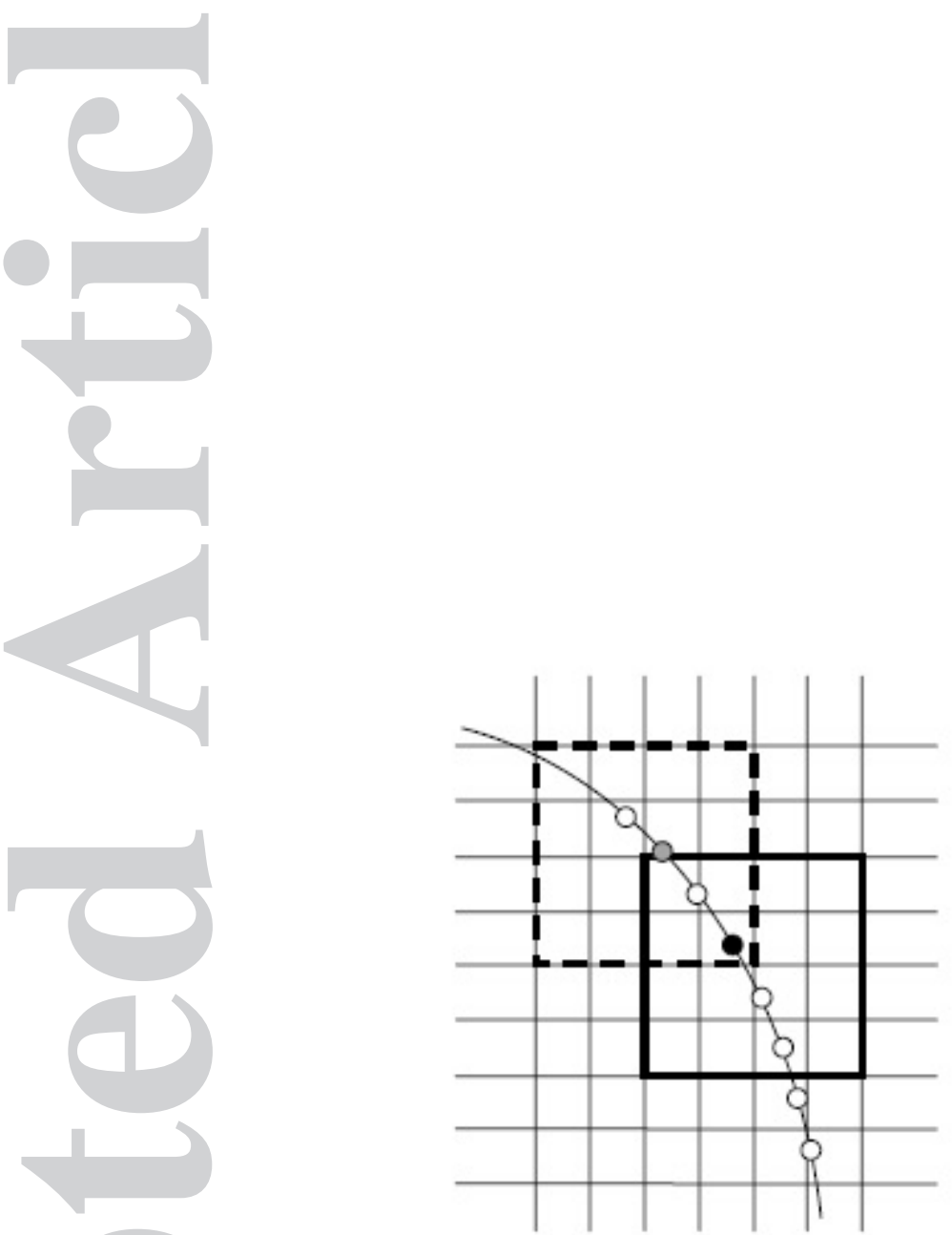

Figure 1. An immersed boundary is shown relative to a two-dimensional Eulerian grid. Several Lagrangian points (circles) are shown on the immersed boundary. The $4 \times 4$ support cage for the gray Lagrangian point is outlined by the dashed square, similarly the support cage for the dark Lagrangian point is outlined by the solid square.

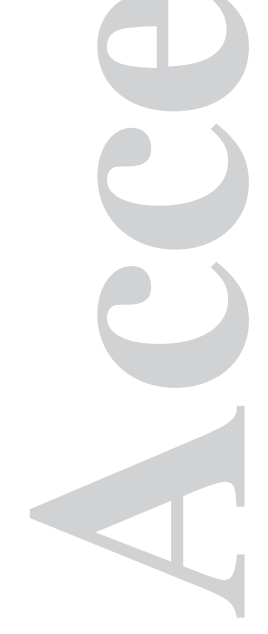

This article is protected by copyright. All rights reserved. 

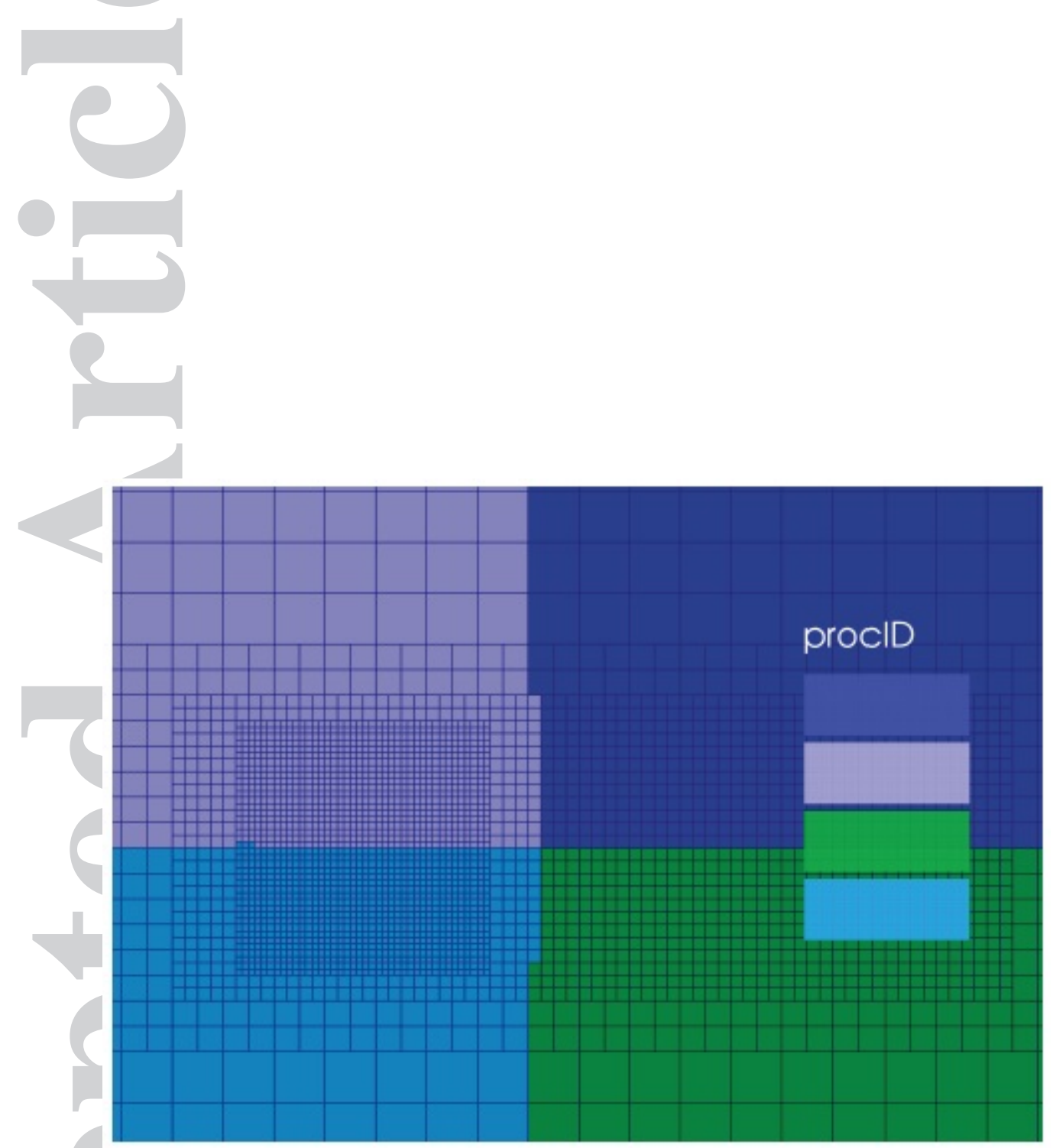

Figure 2. Decomposition of a local refined mesh on four processors

This article is protected by copyright. All rights reserved. 


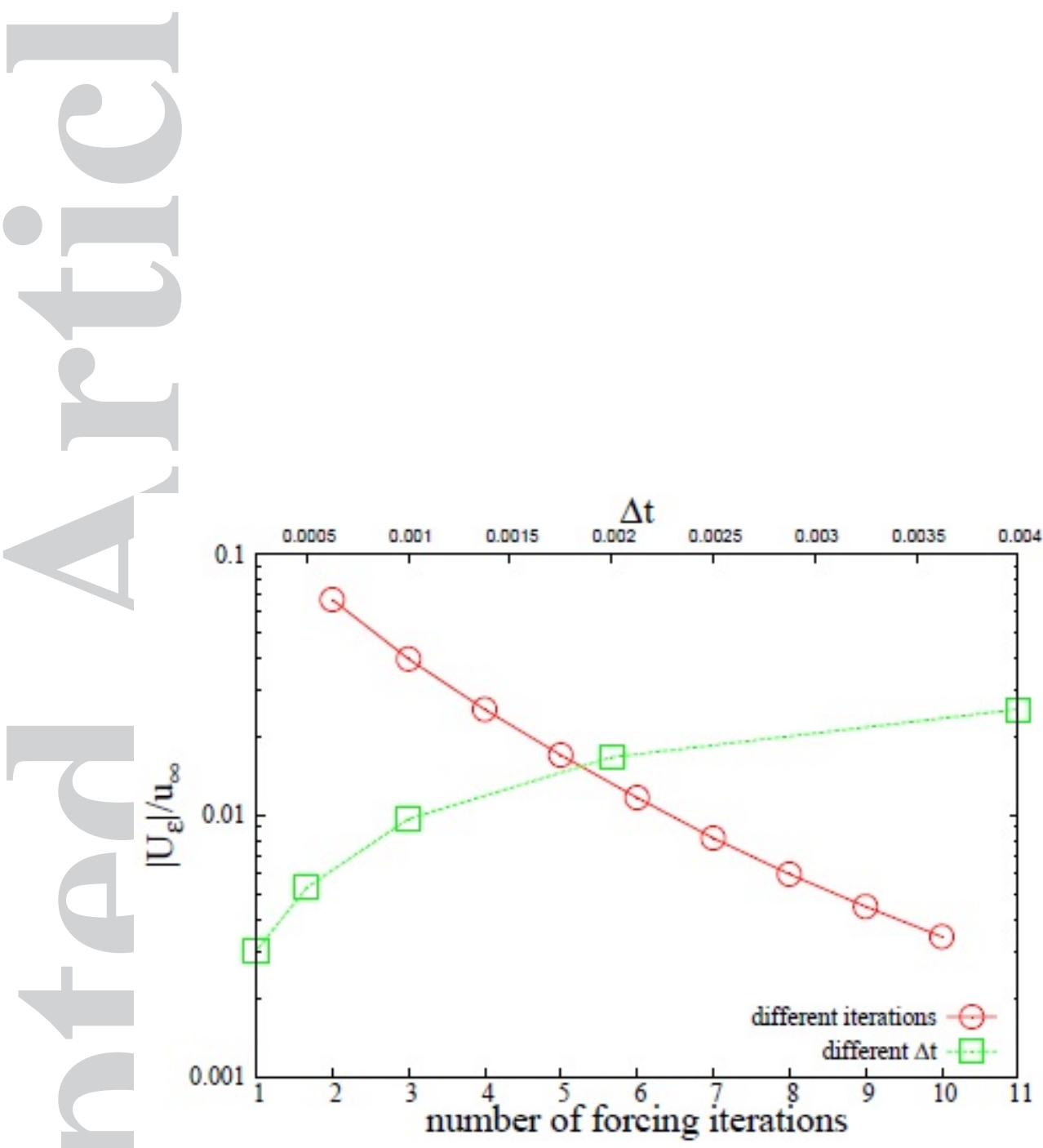

Figure 3. maximum relative error of $|U|$ among all Lagrangian points; circles show error by fixing the time step length as $\Delta t=0.004$ to evaluate the error for different number of forcing iterations, squares show error by fixing the number of forcing iterations as 4 to evaluate the error for different time steps; $R e=10$ and $\Delta x=1 / 50$.

This article is protected by copyright. All rights reserved. 


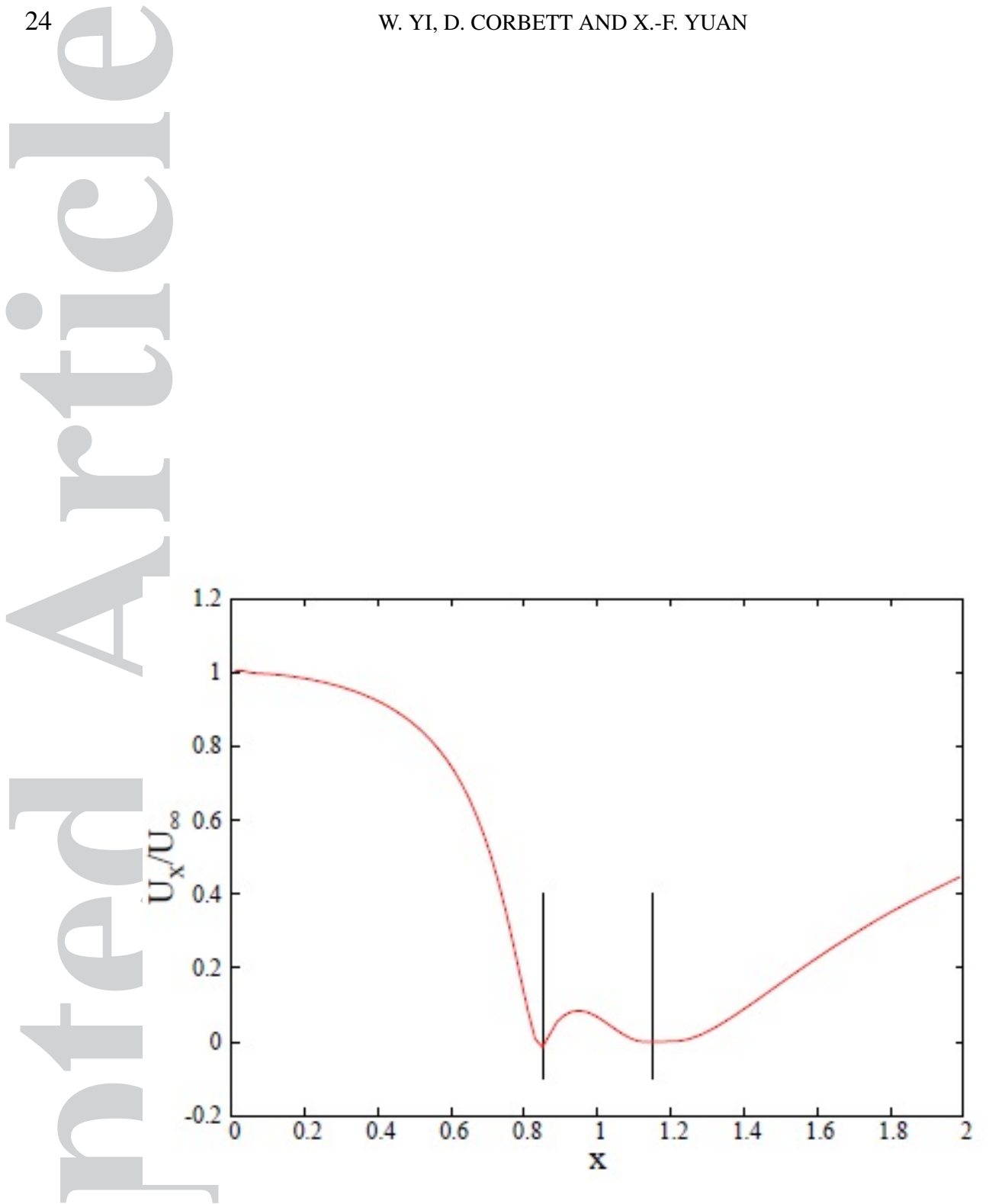

Figure 4. $U_{x}$ profile along the horizontal line $y=1$; simulation is with two forcing steps and the initial prediction of boundary forces on Lagrangian points are from values of last time step; $R e=10, \Delta x=1 / 50$ and $\Delta t=0.004$.

This article is protected by copyright. All rights reserved. 


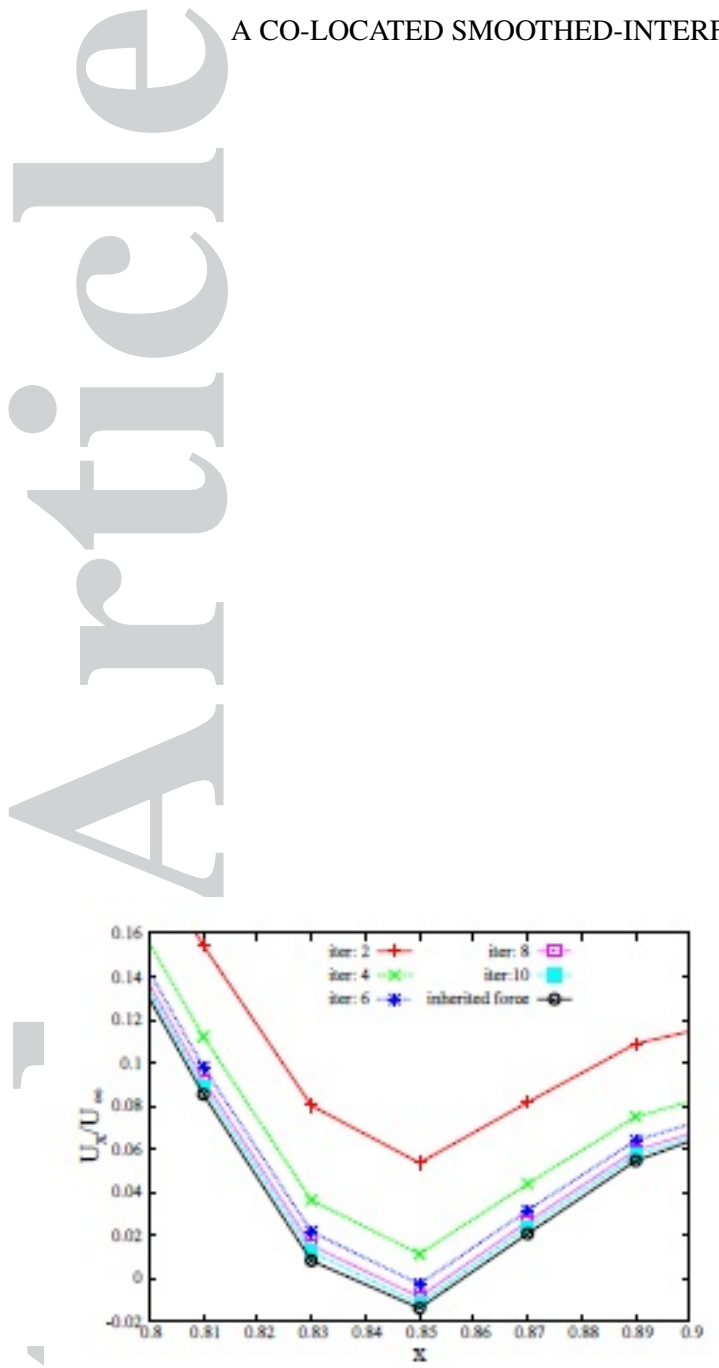

(a) fixed time step as $\Delta t=0.004$

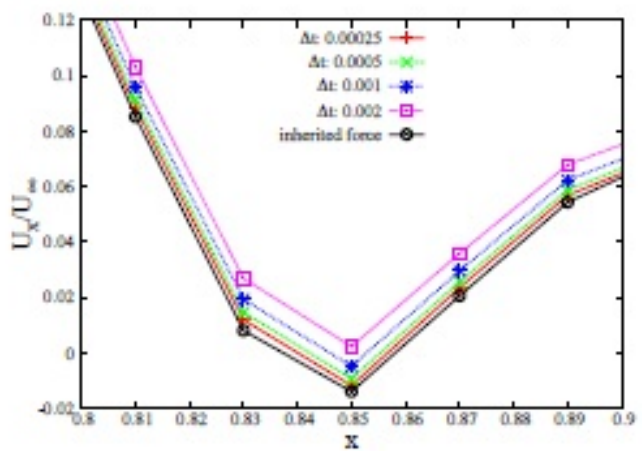

(b) fixed number of iterations as 4

Figure 5. $U_{x}$ profile along the horizontal line $y=1 ; R e=10, \Delta x=1 / 50$

This article is protected by copyright. All rights reserved. 


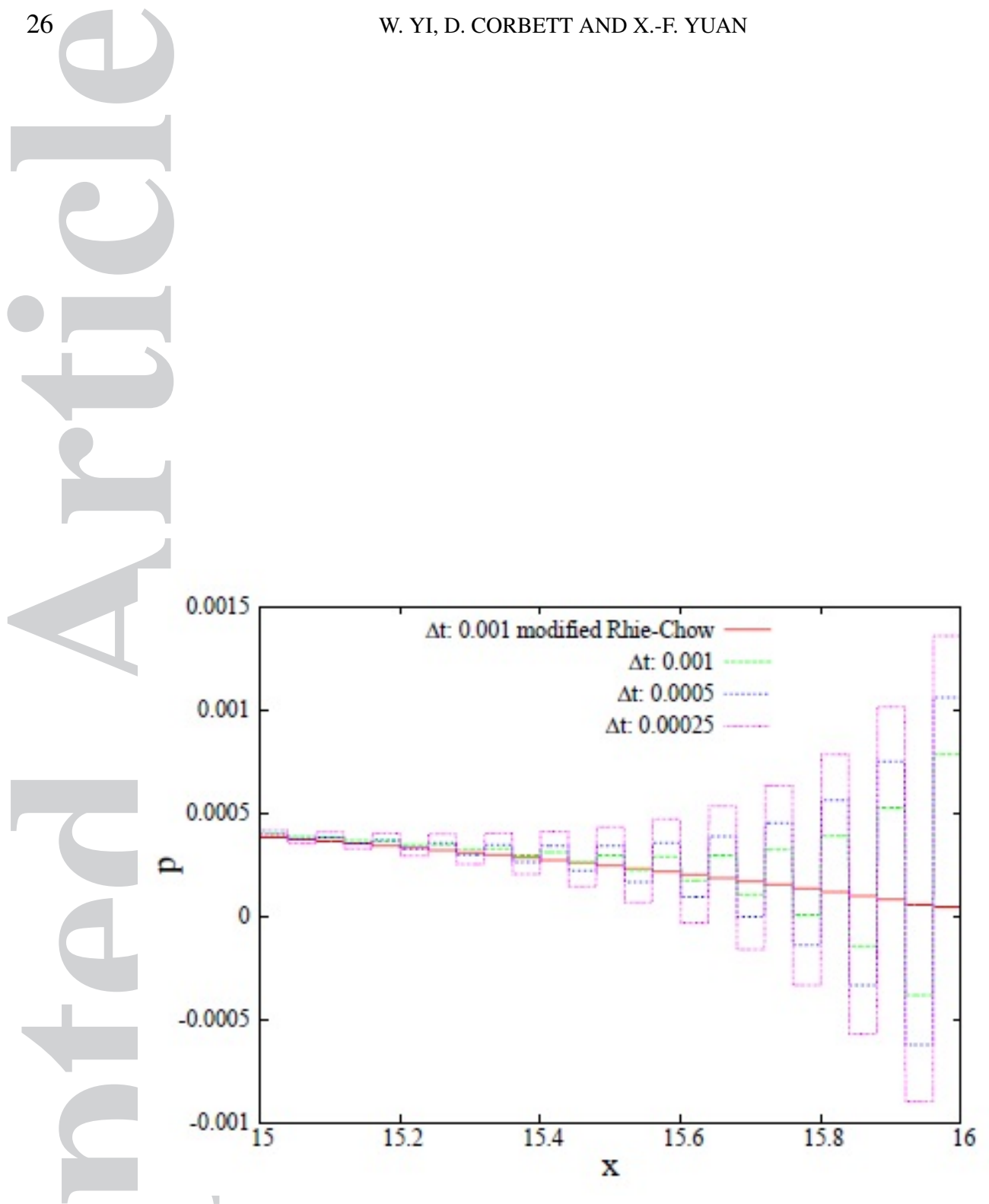

Figure 6. pressure profile along the horizonal line $y=8$; simulation results on a local refined grid from the modified-Rhie-Chow interpolation is compared with the original Rhie-Chow interpolation; $R e=40$, $\Delta x_{\min }=1 / 200$ 


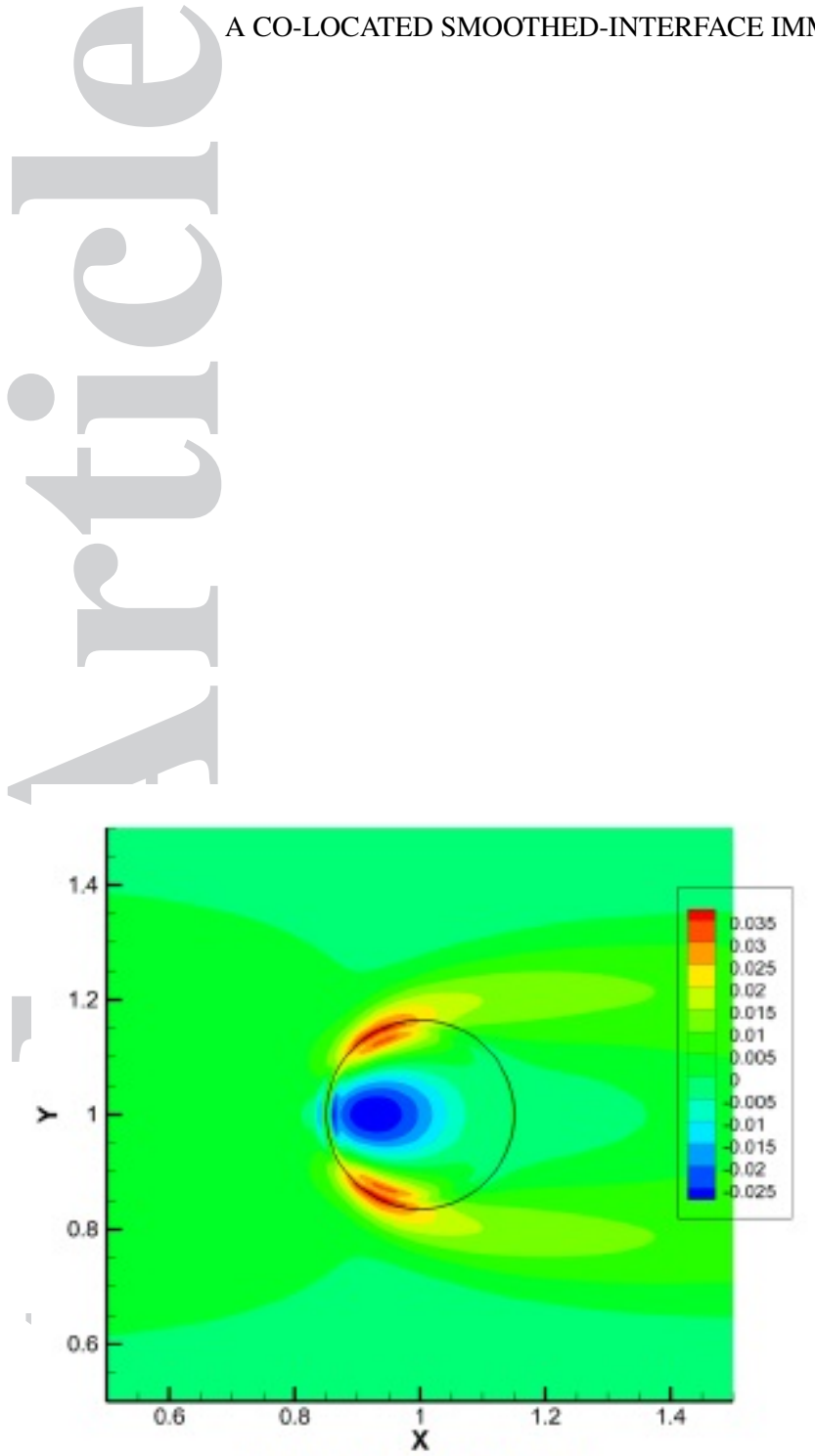

(a) modified-Rhie-Chow

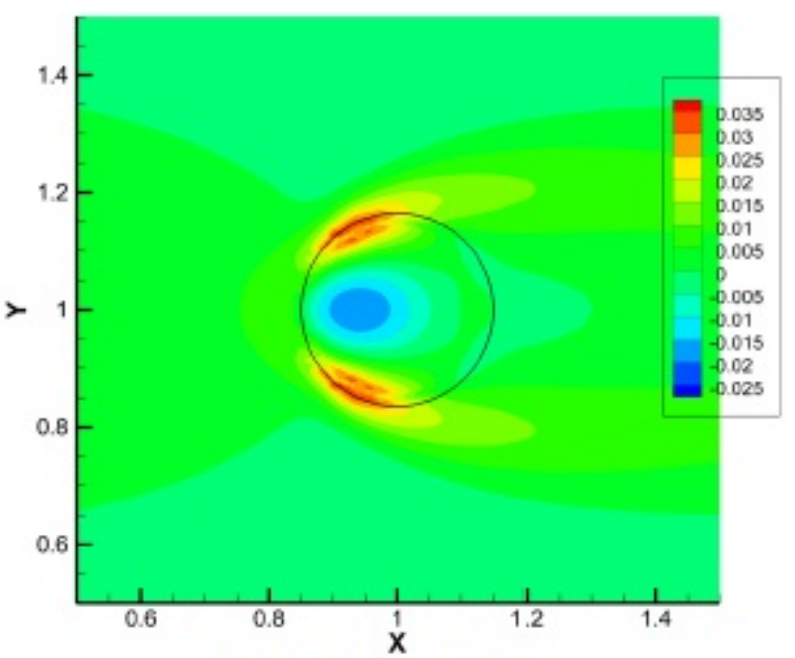

(b) improved-Rhie-Chow

Figure 7. $U_{x}$ difference between simulation result with mesh $800 \times 800$ and $400 \times 400$; Courant No. $\approx 0.4$, $R e=40$.

This article is protected by copyright. All rights reserved. 


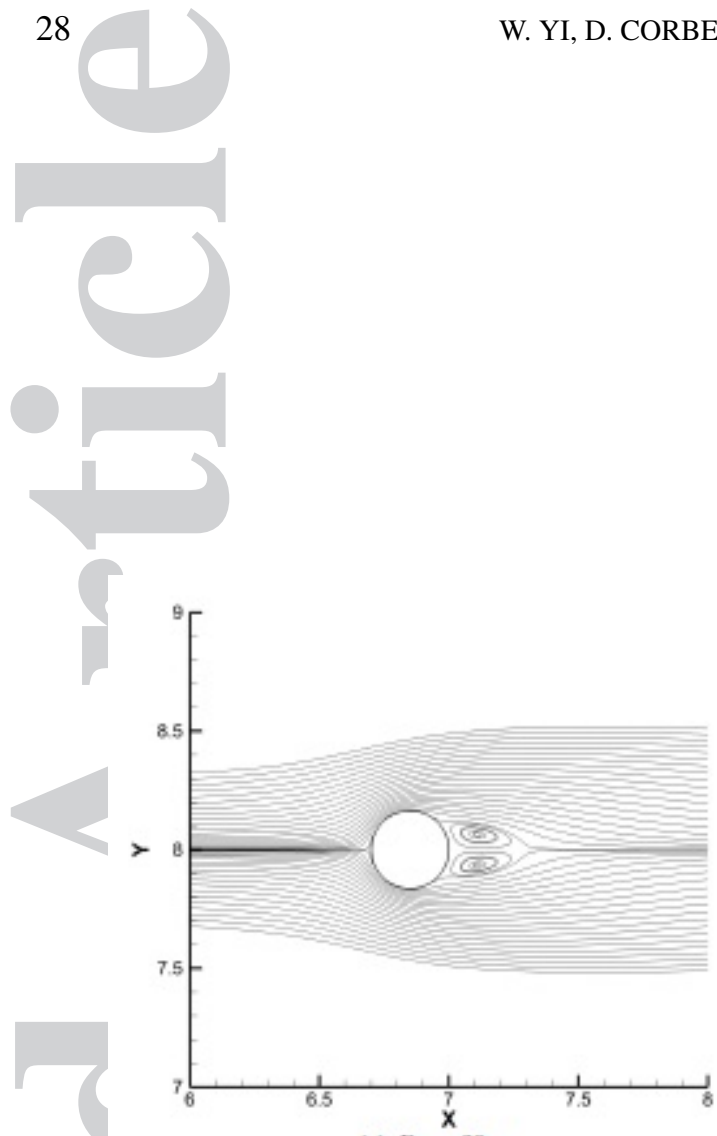

(a) $\operatorname{Re}=20$

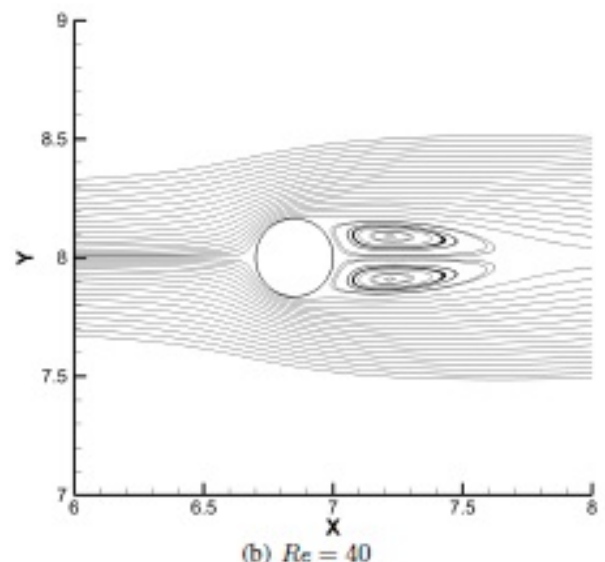

(b) $R e=40$

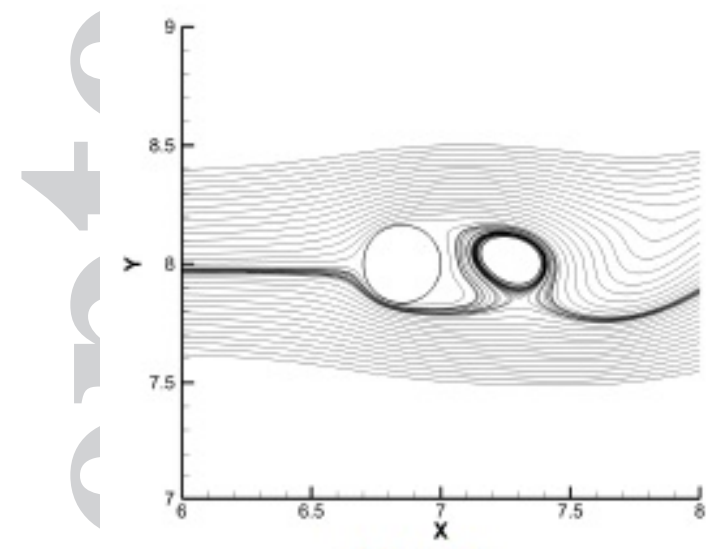

(c) $\operatorname{Re}=100$

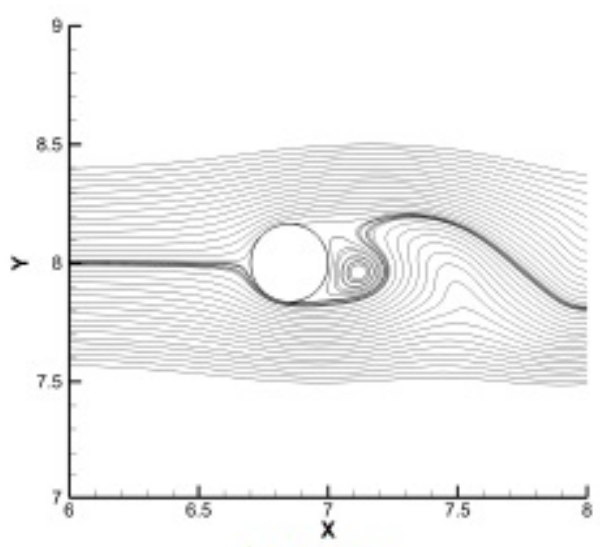

(d) $\operatorname{Re}=200$

Figure 8. Streamline of flow past a cylinder for different Reynolds number with improved-Rhie-Chow solver;

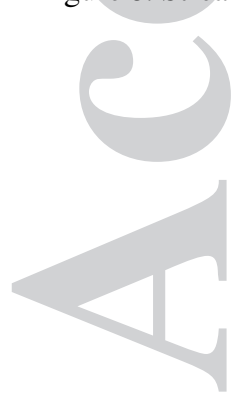
$\Delta x_{\min }=D / 120, t=50$. 


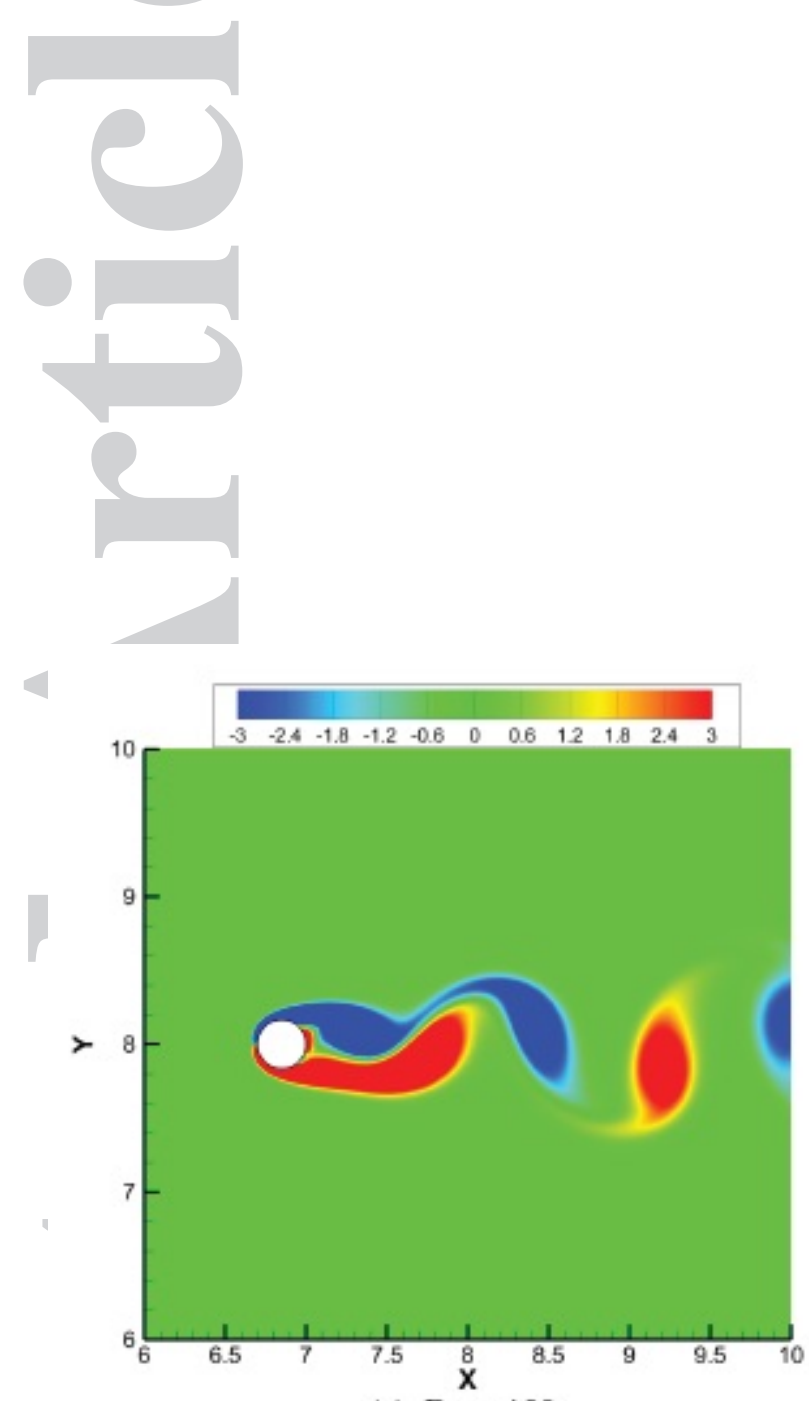

(a) $R e=100$

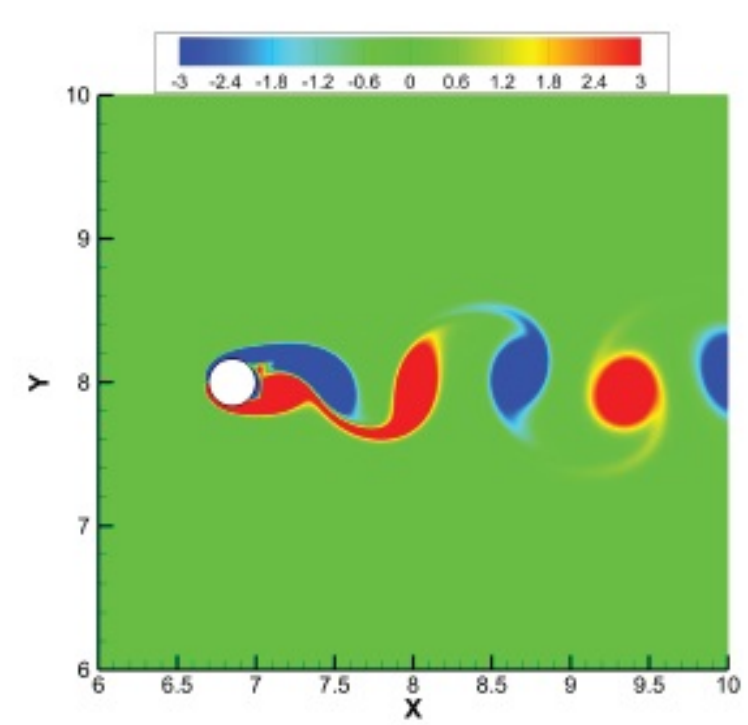

(b) $R e=200$

Figure 9. $z$-direction vorticity with improved-Rhie-Chow solver; $\Delta x_{\min }=1 / 400, t=50$.

This article is protected by copyright. All rights reserved. 


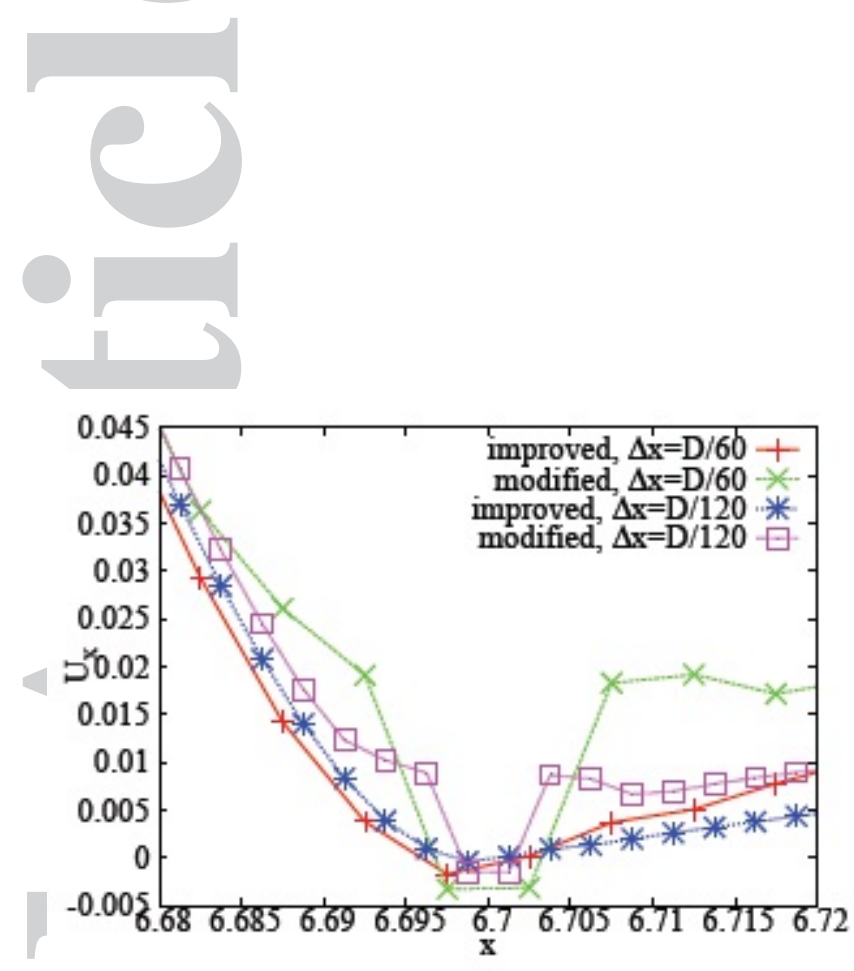

(a) $U_{x}$ along $y=8$

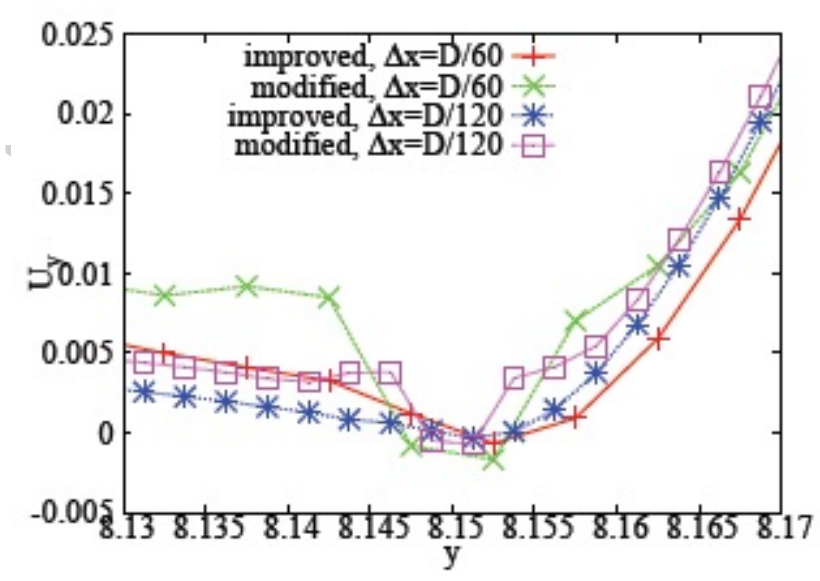

(c) $U_{y}$ along $x=6.85$

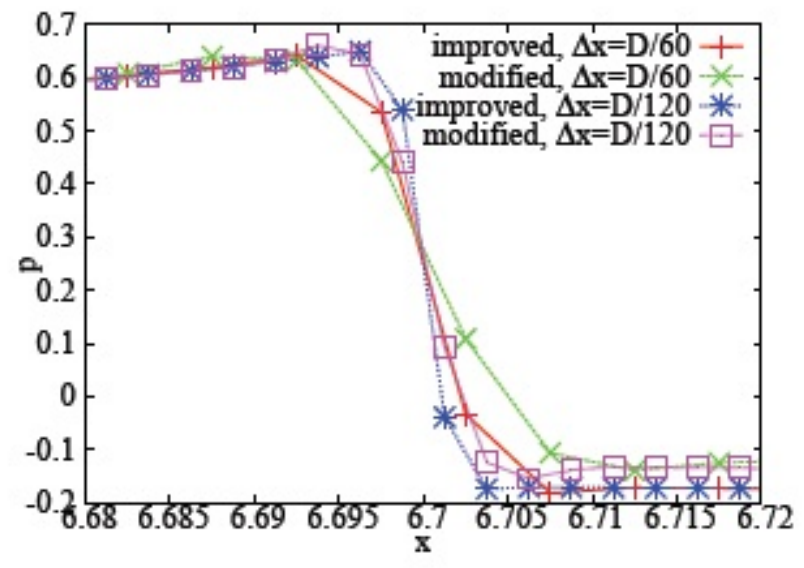

(b) $p$ along $y=8$

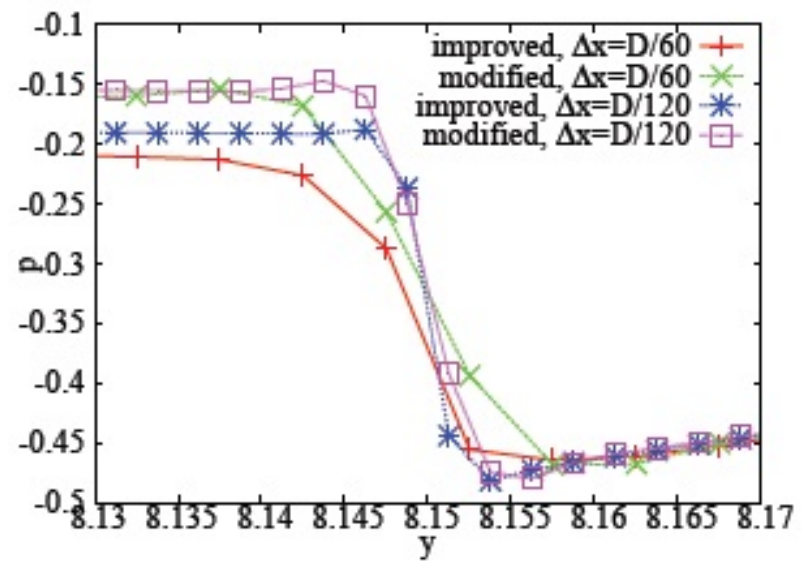

(d) $p$ along $x=6.85$

Figure 10. velocity and pressure profile at the front and up point of the cylinder for $R e=20$

This article is protected by copyright. All rights reserved. 


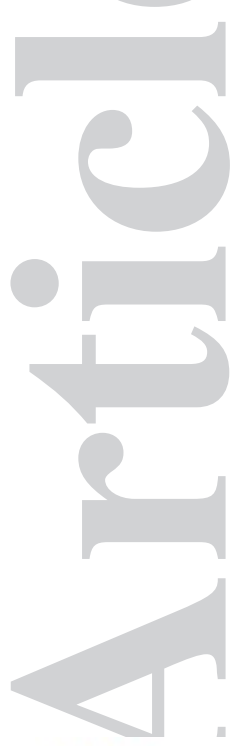

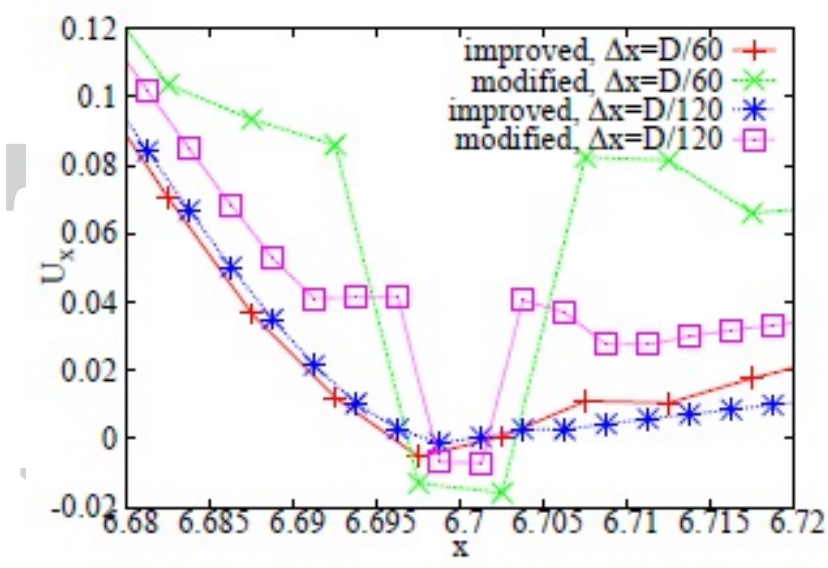

(a) $U_{x}$ along $y=8$

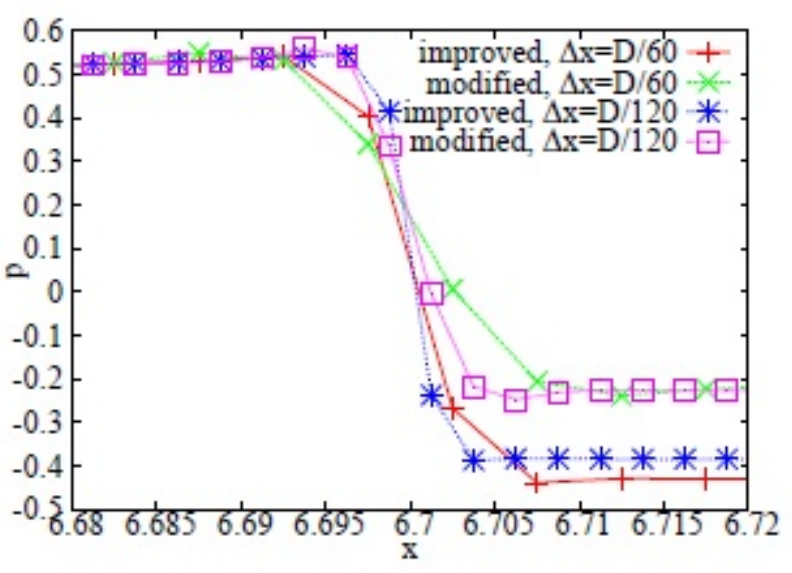

(b) $p$ along $y=8$

Figure 11. velocity and pressure profile at the front point of the cylinder for $R e=100$

This article is protected by copyright. All rights reserved. 


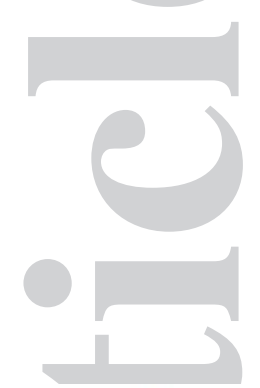

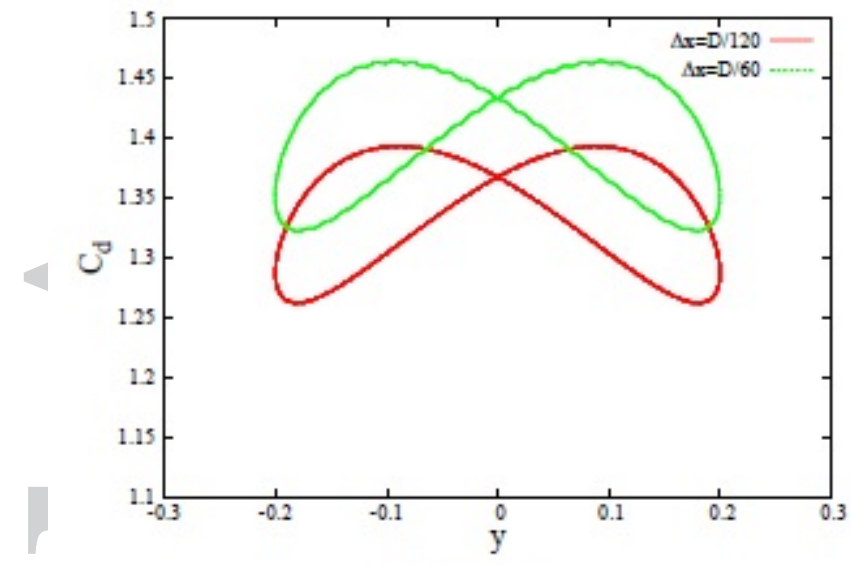

(a) $C_{d}$ (IB4)

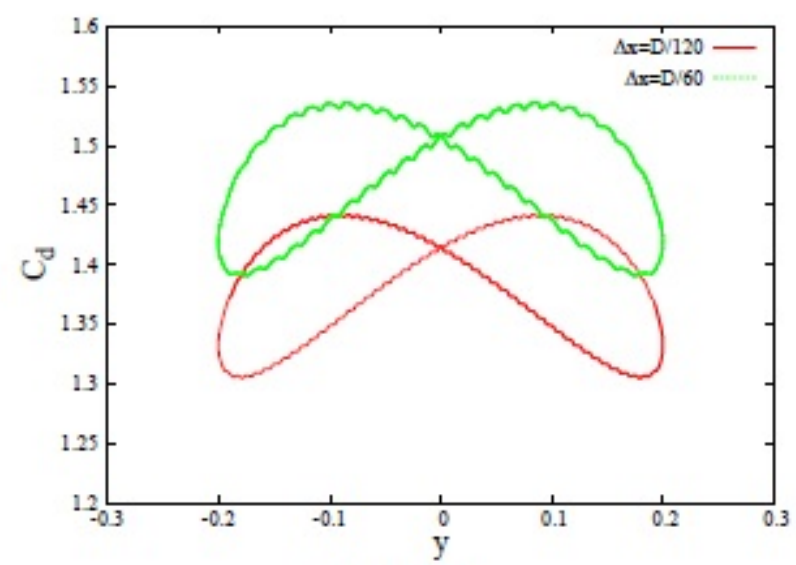

(c) $C_{d}$ (IB3)

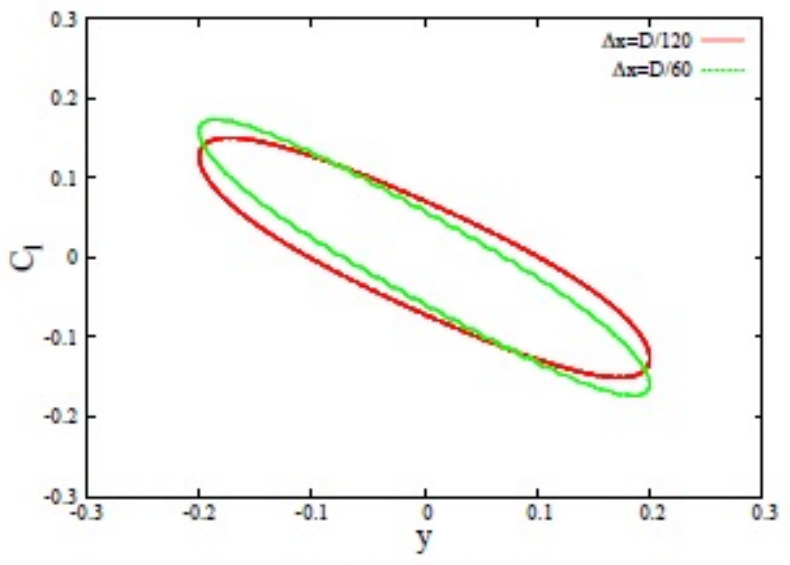

(b) $C_{l}$ (IB4)

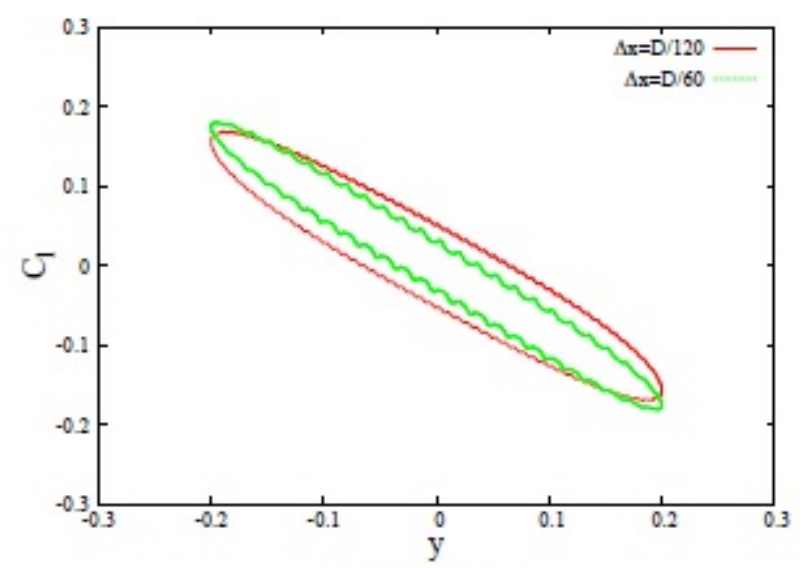

(d) $C_{l}$ (IB3)

Figure 12. drag and lift coefficient for an oscillatory cylinder in free stream with modified-Rhie-Chow interpolation; $R e=185, f_{e} / f_{o}=0.8$, Courant No. $\approx 0.4$.

This article is protected by copyright. All rights reserved. 


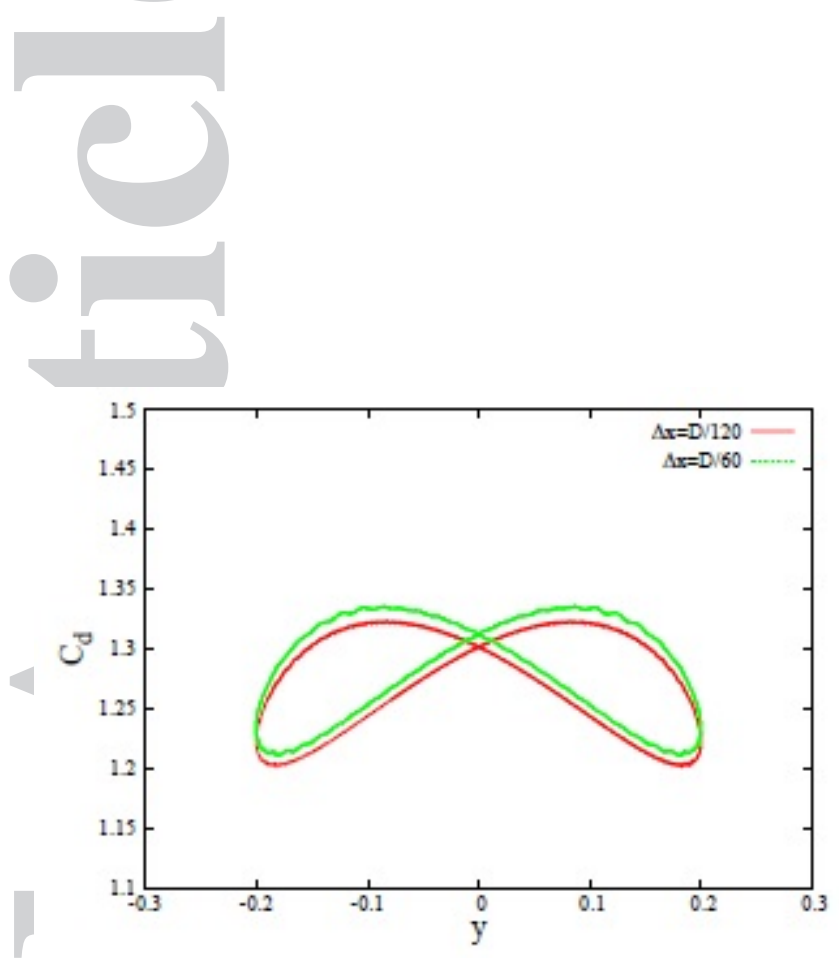

(a) $C_{d}$ (IB4)

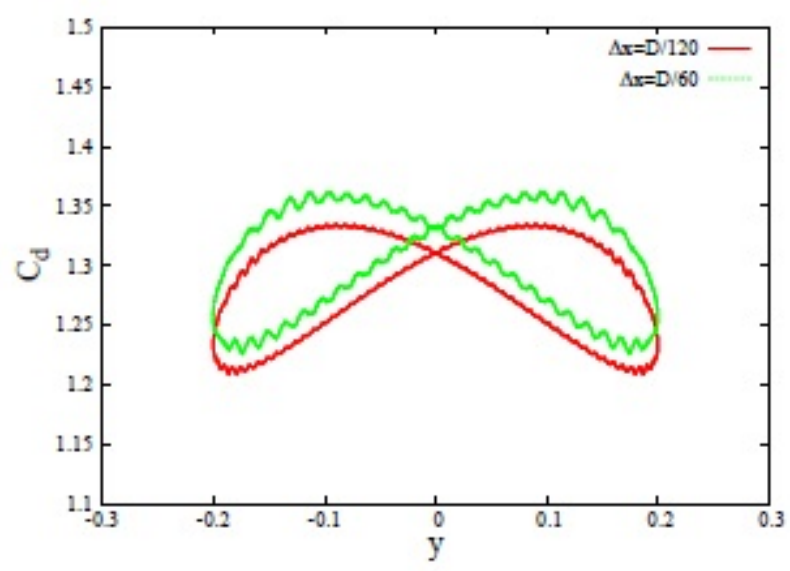

(c) $C_{d}$ (IB3)

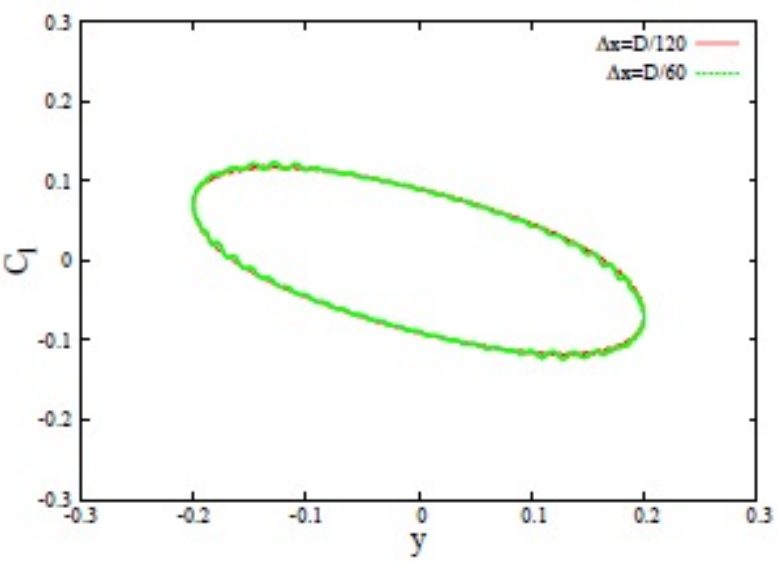

(b) $C_{l}$ (IB4)

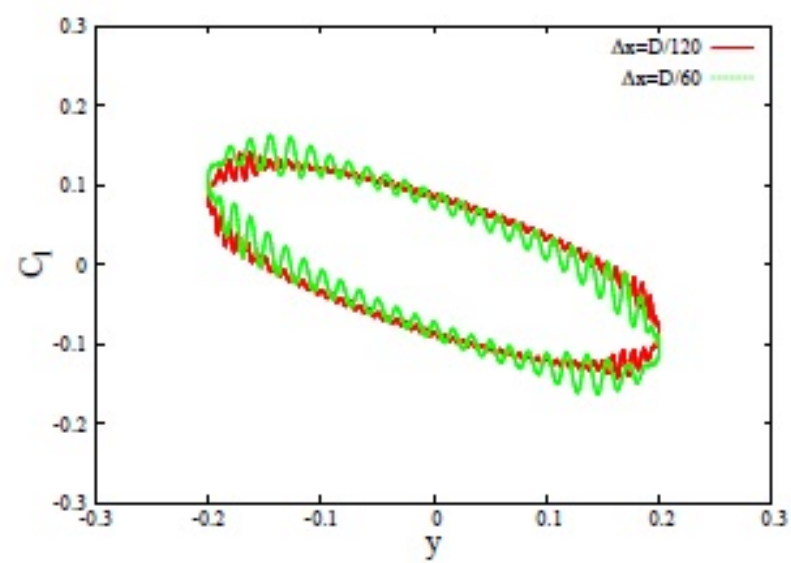

(d) $C_{l}$ (IB3)

Figure 13. drag and lift coefficient for an oscillatory cylinder in free stream with improved-Rhie-Chow interpolation; $R e=185, f_{e} / f_{o}=0.8$, Courant No. $\approx 0.4$.

This article is protected by copyright. All rights reserved. 


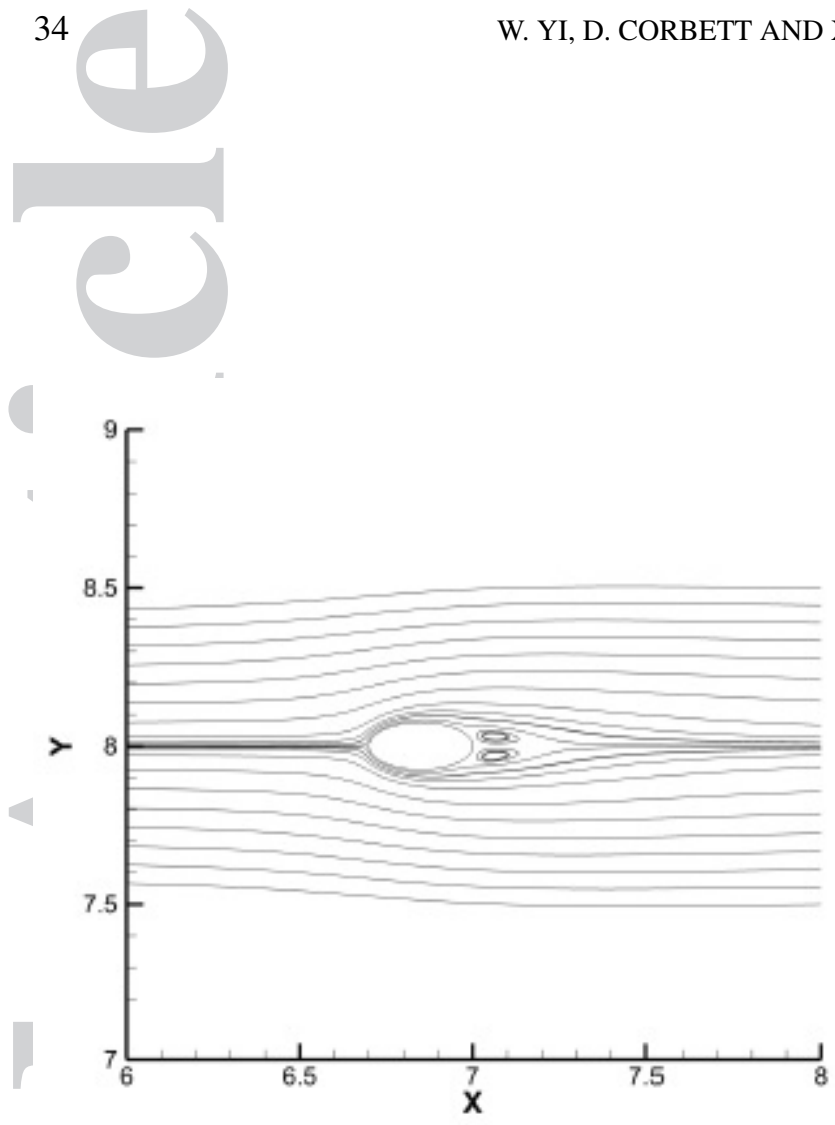

(a) $\alpha=0$

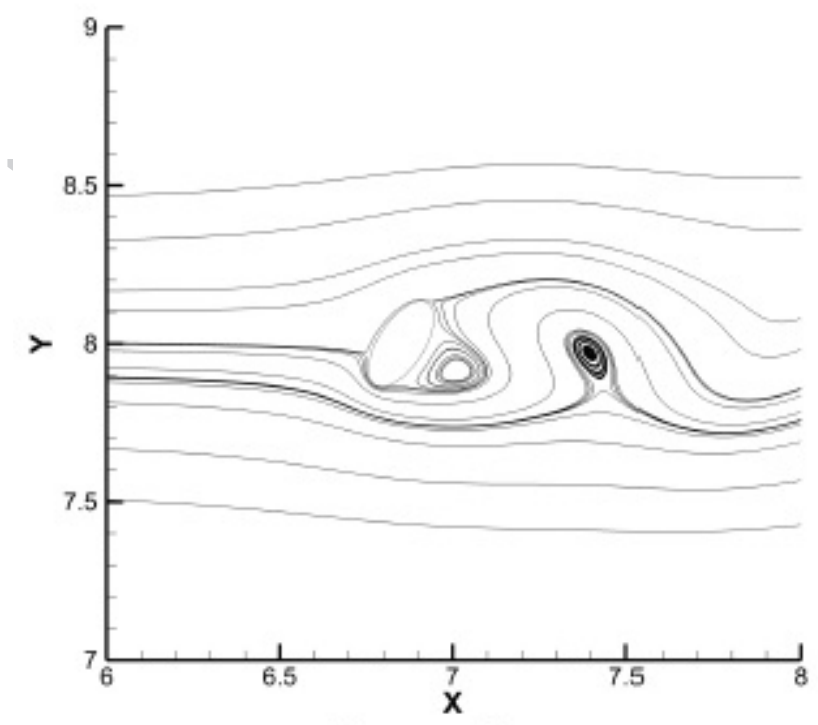

(c) $\alpha=\pi / 3$

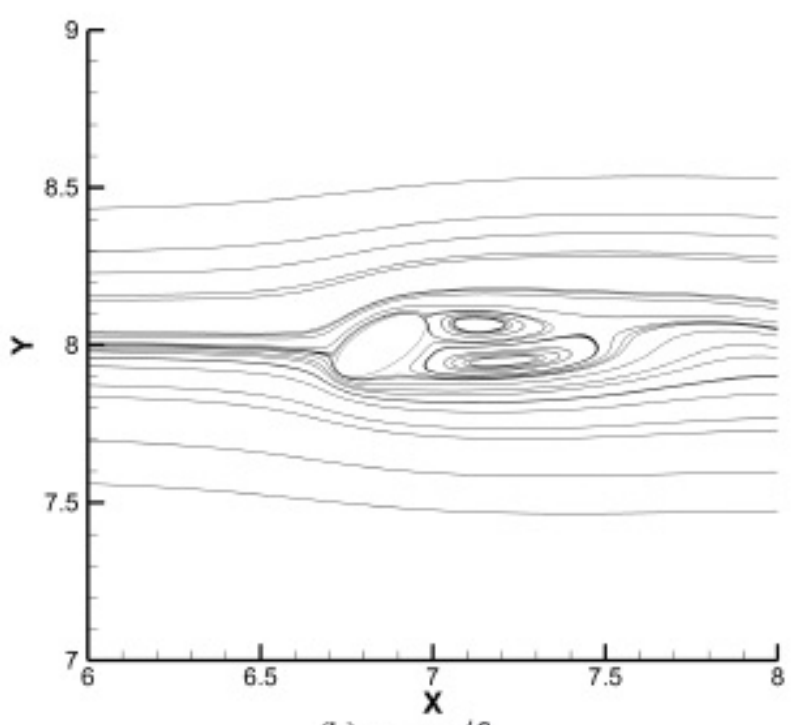

(b) $\alpha=\pi / 6$

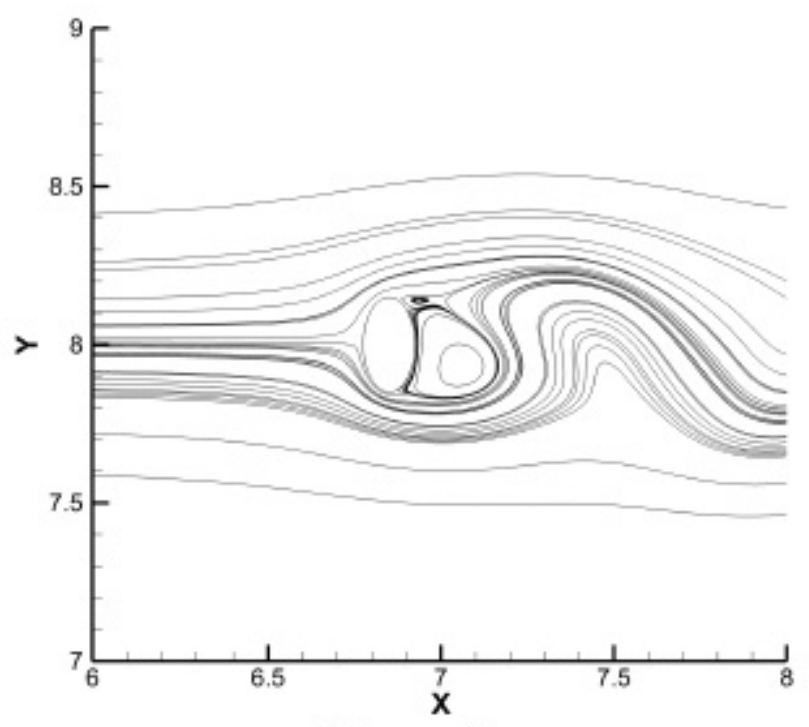

(d) $\alpha=\pi / 2$

Figure 14. Streamlines of flow past a stationary elliptical cylinder with different attack angles at $T=200 \mathrm{~s}$

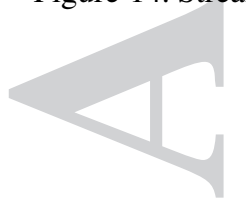
when $R e=40$.

This article is protected by copyright. All rights reserved. 


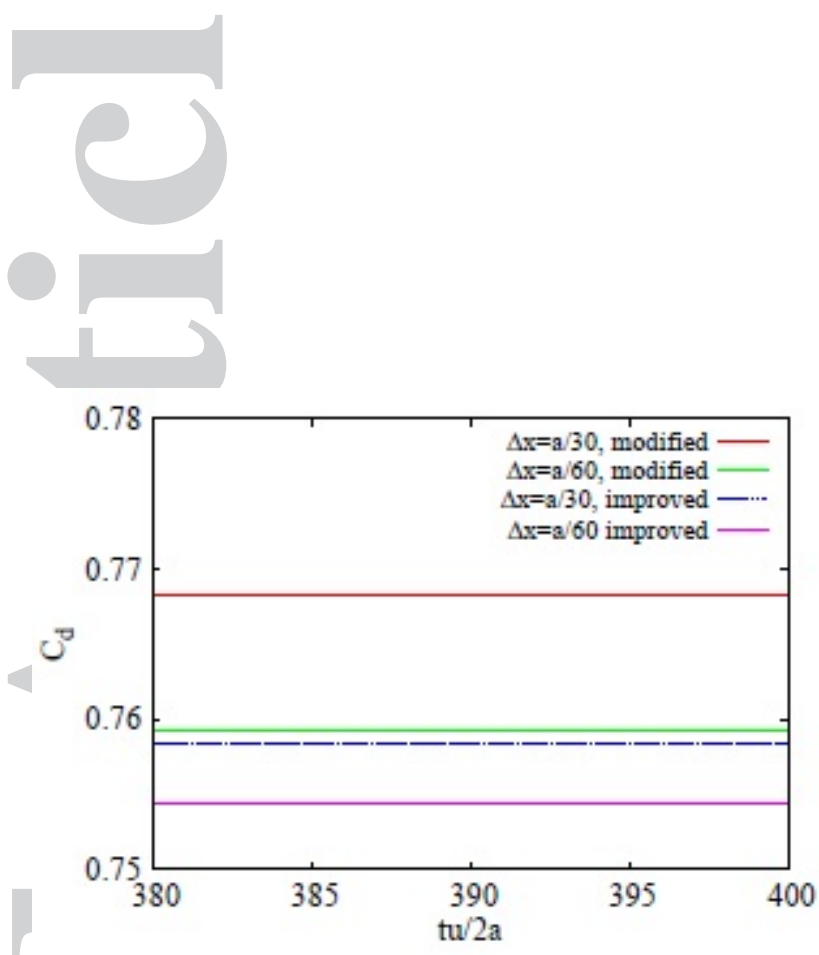

(a) $\alpha=0$

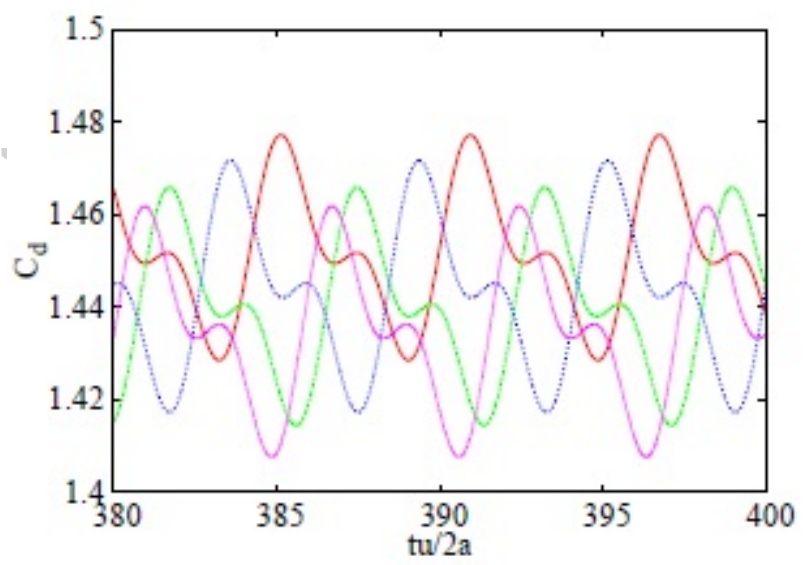

(c) $\alpha=\pi / 3$

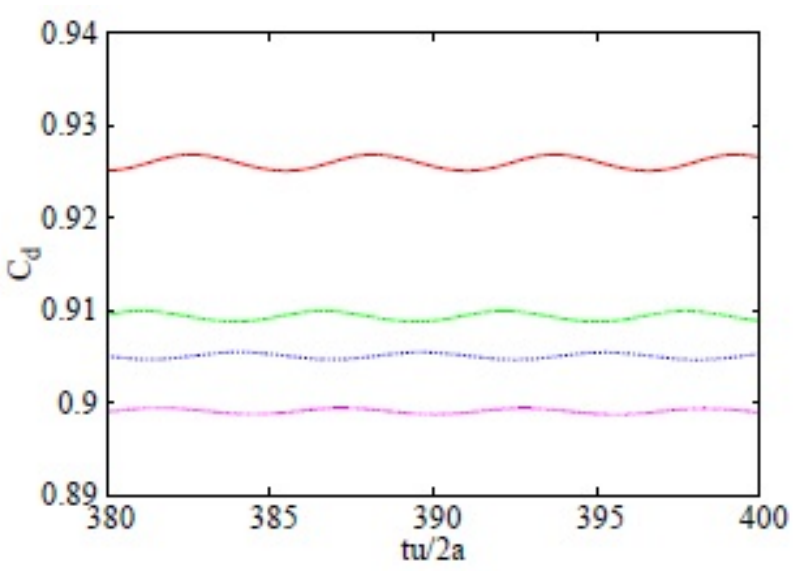

(b) $\alpha=\pi / 6$

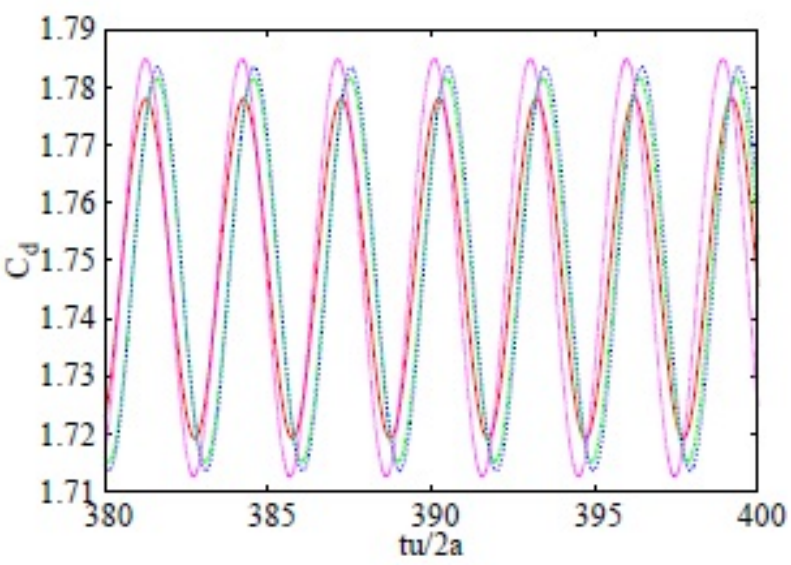

(d) $\alpha=\pi / 2$

Figure 15. Drag coefficients on an elliptical cylinder for different angles of attack when $R e=40$.

This article is protected by copyright. All rights reserved. 


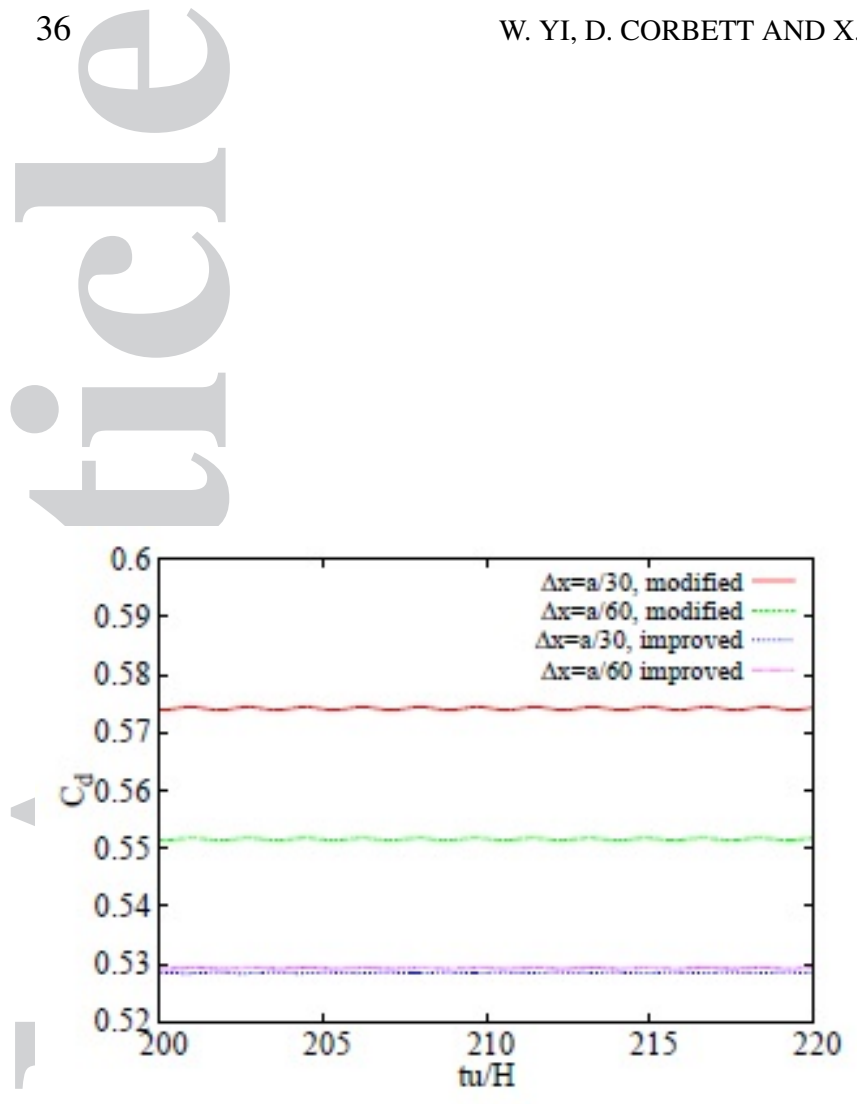

(a) $\alpha=0$

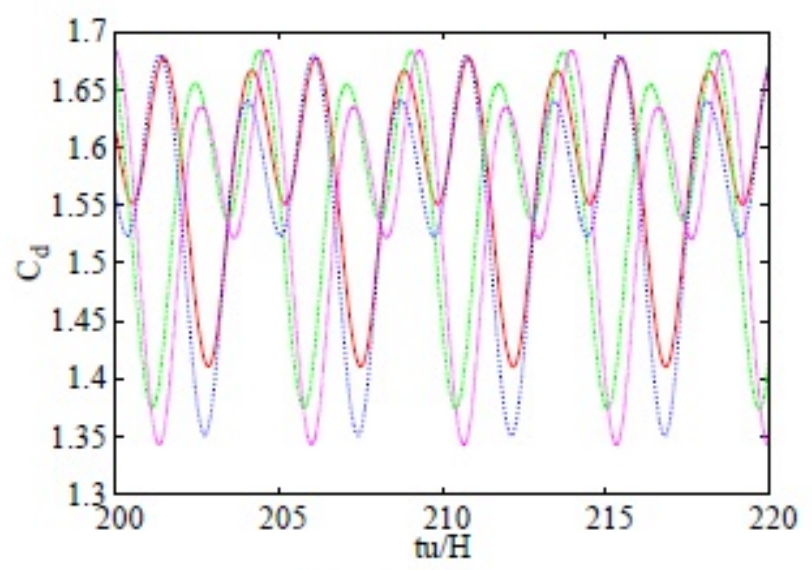

(c) $\alpha=\pi / 3$

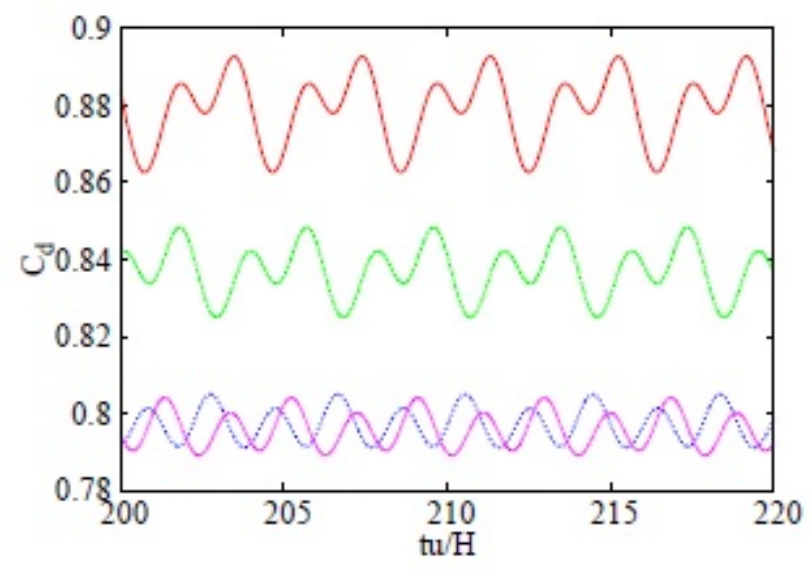

(b) $\alpha=\pi / 6$

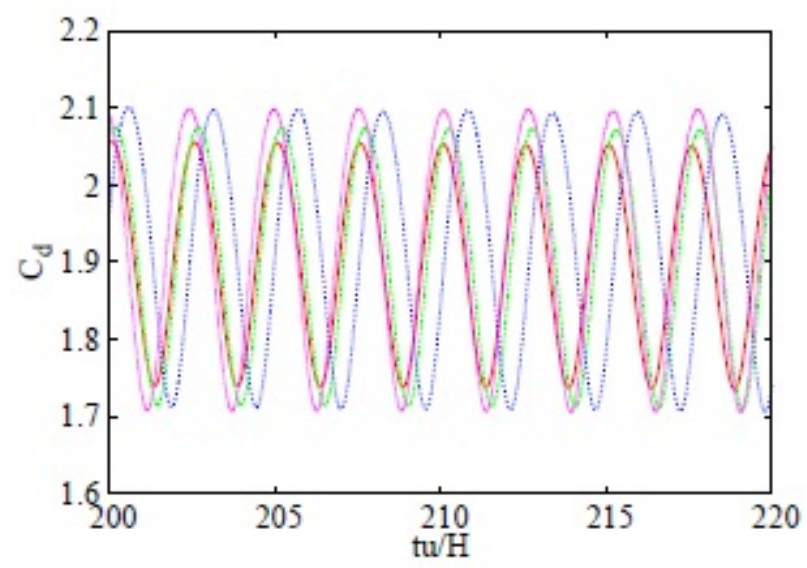

(d) $\alpha=\pi / 2$

Figure 16. Drag coefficients on an elliptical cylinder for different angles of attack when $R e=100$.

This article is protected by copyright. All rights reserved. 

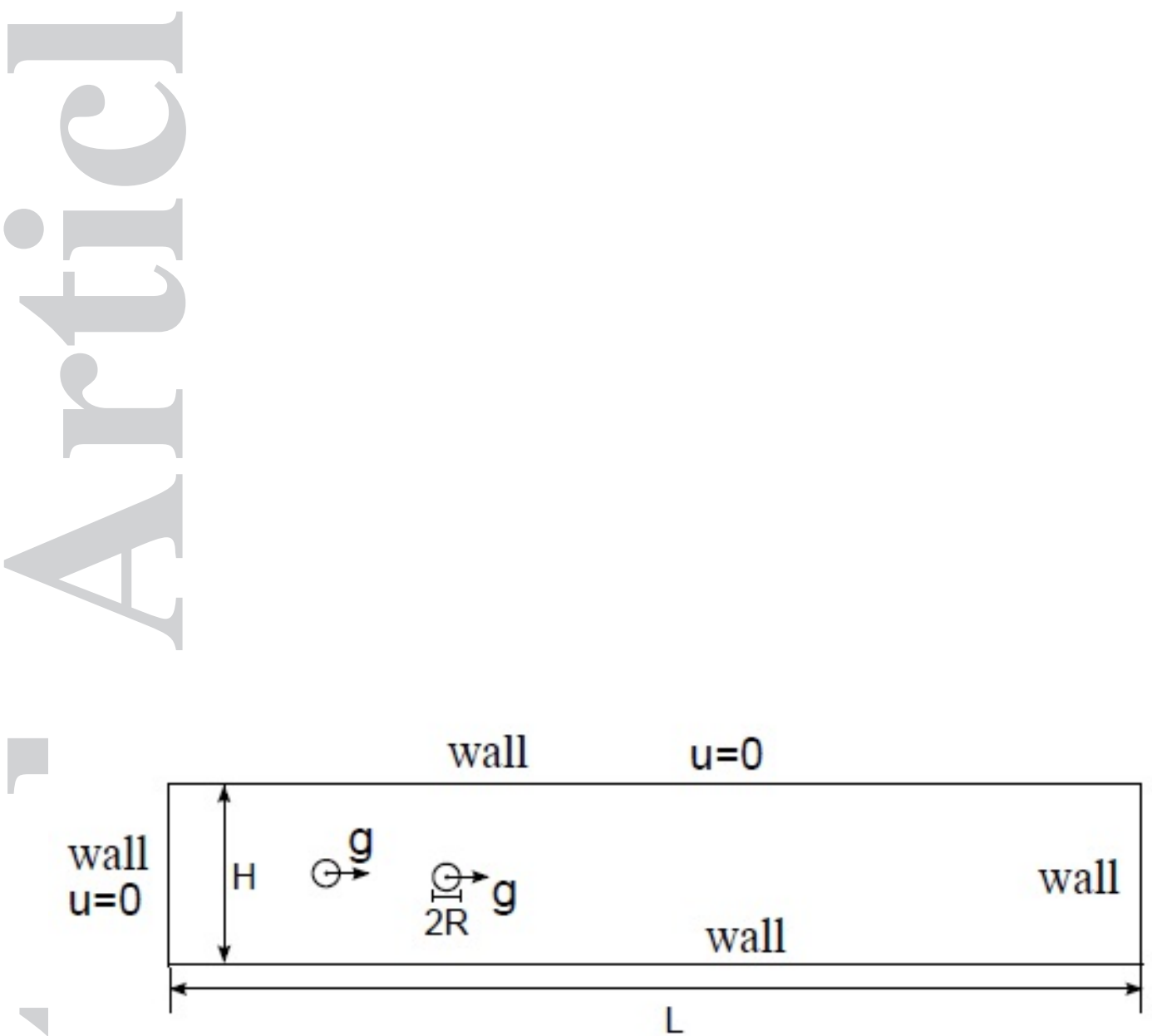

Figure 17. Computational geometry for sedimentation of a pair of cylinders in 2D.

This article is protected by copyright. All rights reserved. 


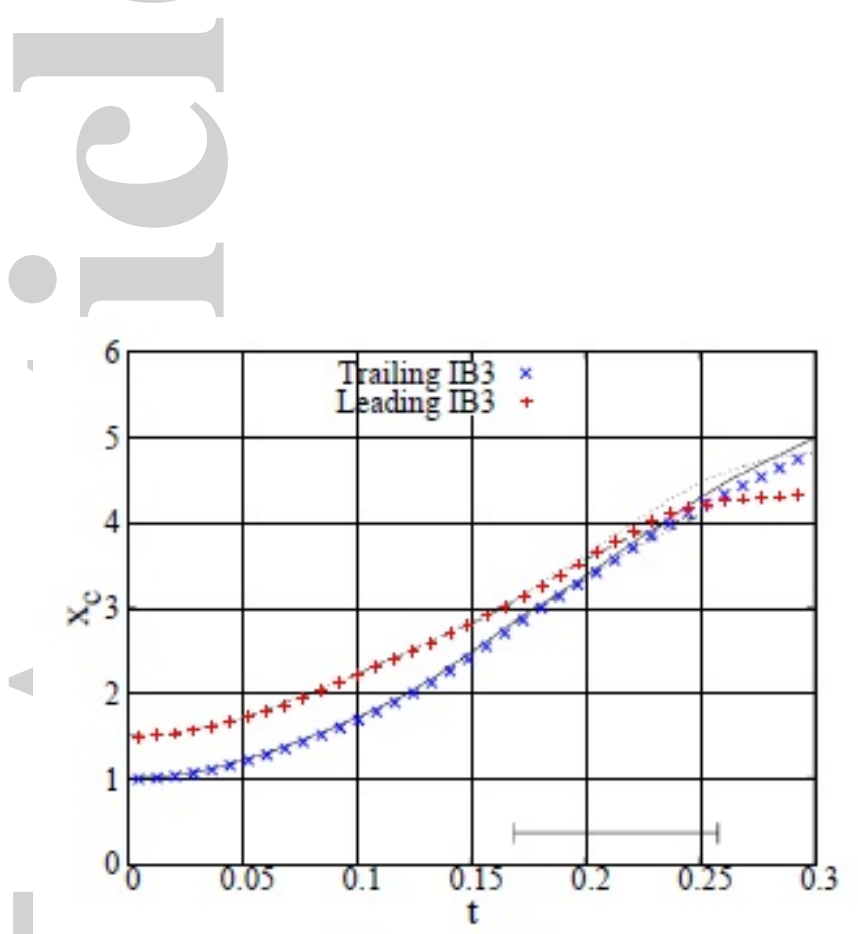

(a) $x$-coordinate

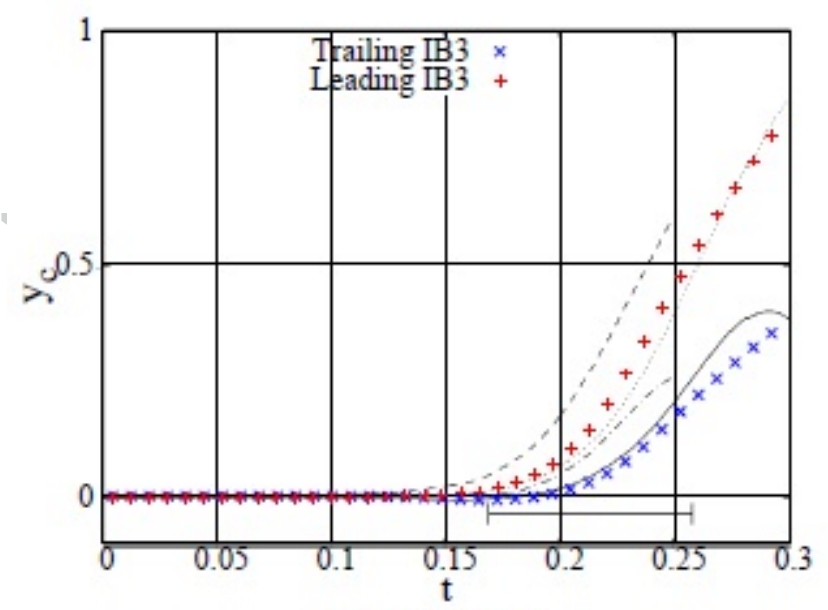

(c) $y$-coordinate

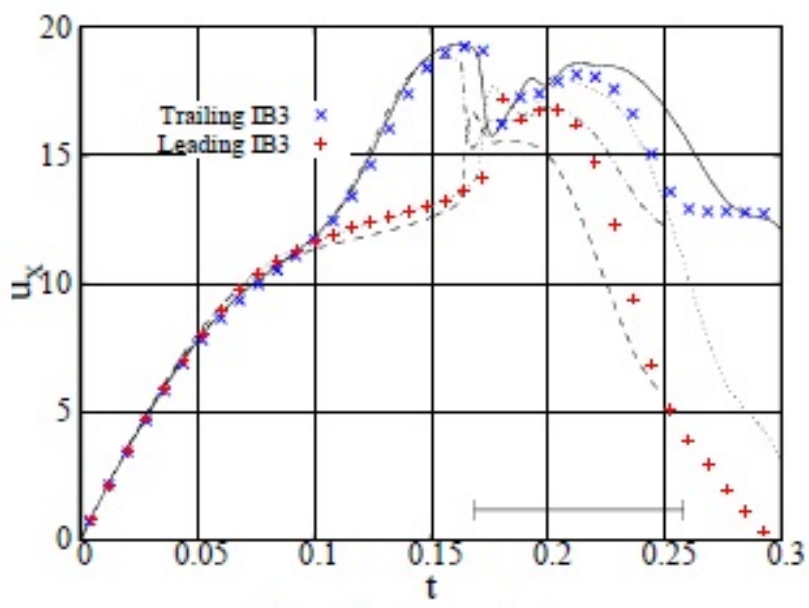

(b) $x$-direction velocity

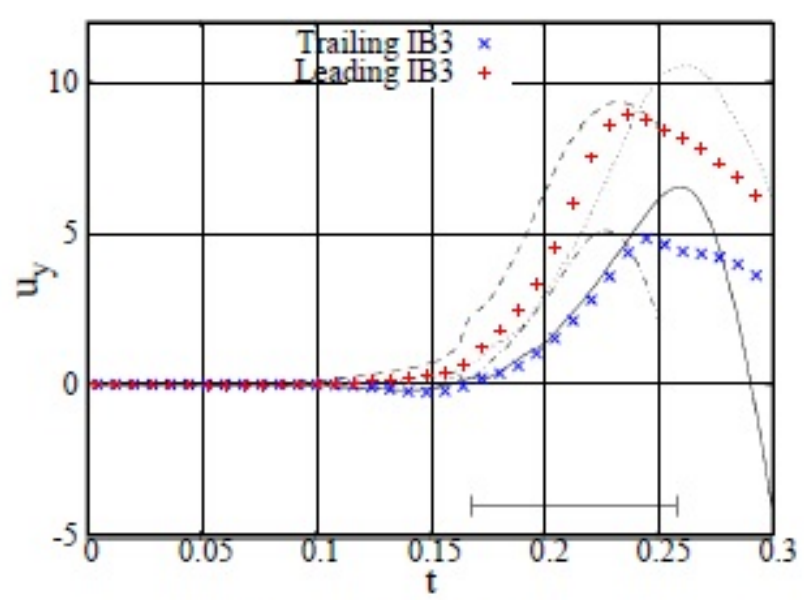

(d) $y$-direction velocity

Figure 18. Displacement and velocity of a pair of cylinders in sedimentation with the improved-Rhie-Chow interpolation. Results are compared with that in [6] using the same time step $(\Delta t=0.0001)$ and grid spacing $(\Delta x=D / 64)$. The gray scale lines are extracted from Figure 6 and Figure 7 in [6], where the solid lines and dots represent profiles of the trailing and leading cylinder respectively by Uhlmann [6], while the dash-dot lines and the dashed lines represent profiles of the trailing and the leading cylinders respectively by Pan [6].

This article is protected by copyright. All rights reserved. 


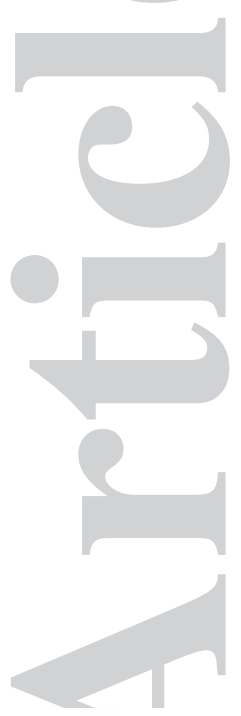

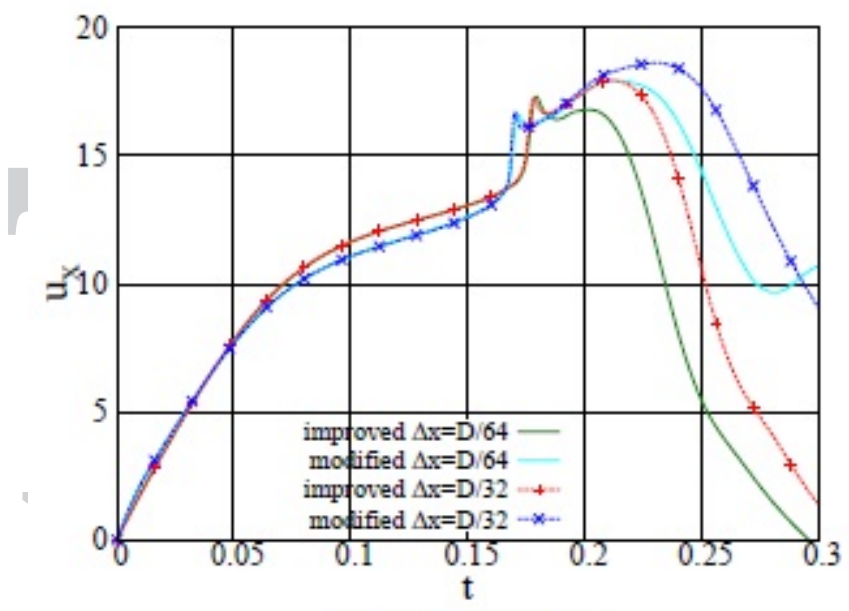

(a) leading cylinder

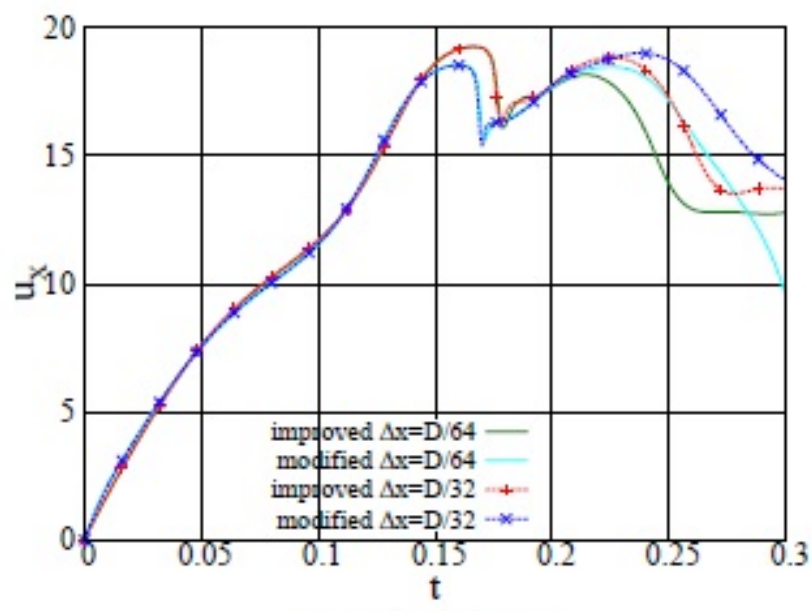

(b) trailing cylinder

Figure 19. Comparison of velocity profiles of two sedimenting cylinders between the modified-Rhie-Chow and the improved-Rhie-Chow interpolation.

This article is protected by copyright. All rights reserved. 


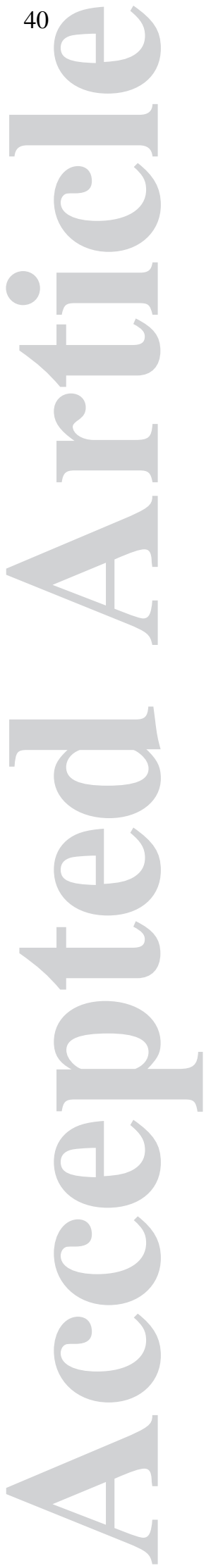

W. YI, D. CORBETT AND X.-F. YUAN

Table I. Relative error for the simulation of a channel flow

\begin{tabular}{lccc}
\hline$H / \Delta x$ & 10 & 20 & 40 \\
$e / \bar{u}$ & $2.5 \times 10^{-3}$ & $6.25 \times 10^{-4}$ & $1.56 \times 10^{-4}$ \\
\hline
\end{tabular}

This article is protected by copyright. All rights reserved. 


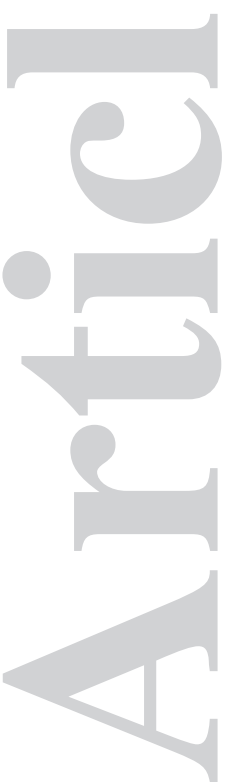

Table II. convergence rate for flow past a cylinder with modified-Rhie-Chow interpolation

\begin{tabular}{|c|c|c|c|c|c|c|c|}
\hline \multicolumn{8}{|c|}{$R e=20$} \\
\hline $\begin{array}{c}\Delta_{x} \\
1 / 25\end{array}$ & $\begin{array}{c}L_{1} \\
0.03954\end{array}$ & $\begin{array}{c}s_{1} \\
138114\end{array}$ & $\begin{array}{c}L_{2} \\
0.05737\end{array}$ & $\begin{array}{c}s_{2} \\
129009\end{array}$ & $\begin{array}{c}L_{\infty} \\
045478\end{array}$ & $\begin{array}{c}s_{\infty} \\
101262\end{array}$ & $\begin{array}{c}C_{d} \\
3.312\end{array}$ \\
\hline $\begin{array}{l}1 / 20 \\
1 / 50\end{array}$ & $\begin{array}{l}0.03954 \\
0.01518\end{array}$ & $\begin{array}{l}1.58114 \\
1.31057\end{array}$ & $\begin{array}{l}0.05 / 3 / \\
0.02346\end{array}$ & 1.27563 & $\begin{array}{l}0.454 / 8 \\
0.22541\end{array}$ & 1.15905 & $\begin{array}{l}5.312 \\
3.020\end{array}$ \\
\hline $1 / 100$ & 0.00612 & 1.64268 & 0.00969 & 0.62114 & 0.10094 & 1.54758 & 2.918 \\
\hline $1 / 200$ & 0.00196 & - & 0.00315 & - & 0.03453 & - & 2.872 \\
\hline \multicolumn{8}{|c|}{$R e=40$} \\
\hline $\begin{array}{l}\Delta_{x} \\
1 / 25\end{array}$ & $\begin{array}{c}L_{1} \\
0.05252\end{array}$ & $\begin{array}{c}s_{1} \\
138782\end{array}$ & $\begin{array}{c}L_{2} \\
007766\end{array}$ & $\begin{array}{c}s_{2} \\
128815\end{array}$ & $\begin{array}{c}L_{\infty} \\
059543\end{array}$ & $\begin{array}{c}s_{\infty} \\
15015\end{array}$ & $\begin{array}{c}C_{d} \\
2577\end{array}$ \\
\hline $1 / 50$ & 0.02007 & 1.25830 & 0.03180 & 1.23500 & 0.29462 & 1.09408 & 2.263 \\
\hline $1 / 100$ & 0.00839 & 1.58840 & 0.01351 & 1.58603 & 0.13801 & 1.53423 & 2.162 \\
\hline $1 / 200$ & 0.00279 & - & 0.00450 & - & 0.04765 & - & 2.115 \\
\hline
\end{tabular}

This article is protected by copyright. All rights reserved. 


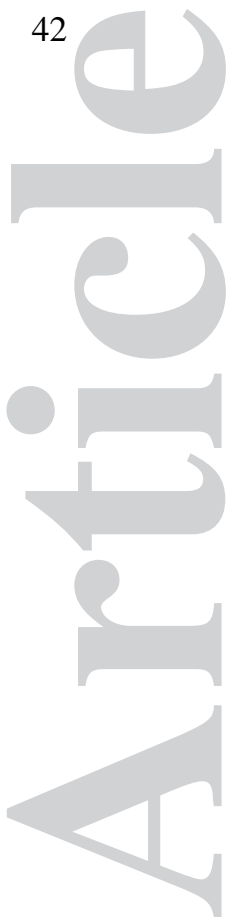

W. YI, D. CORBETT AND X.-F. YUAN

Table III. convergence rate for flow past a cylinder with improved-Rhie-Chow interpolation

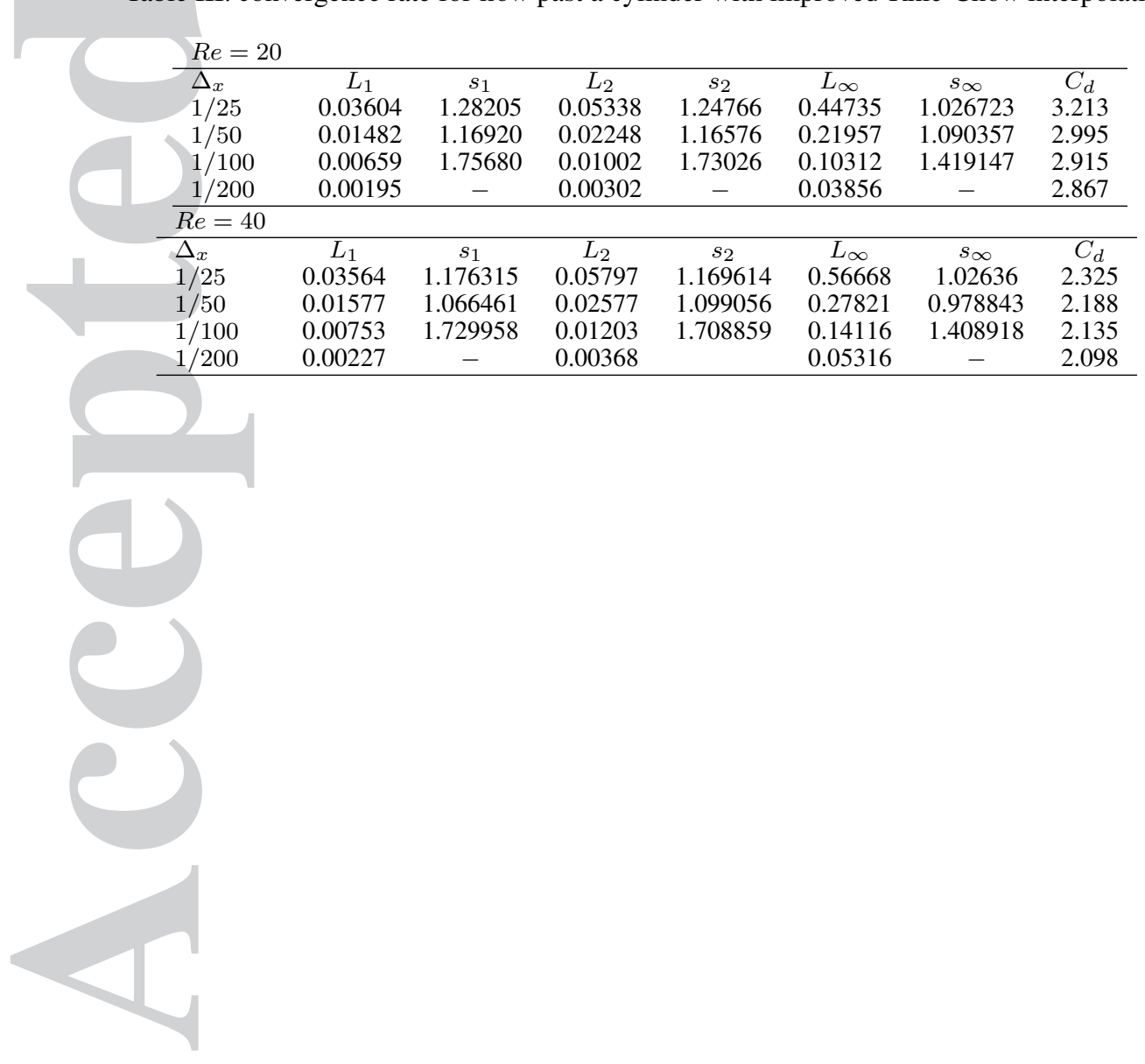

This article is protected by copyright. All rights reserved. 
Table IV. drag coefficient for flow past a stationary cylinder

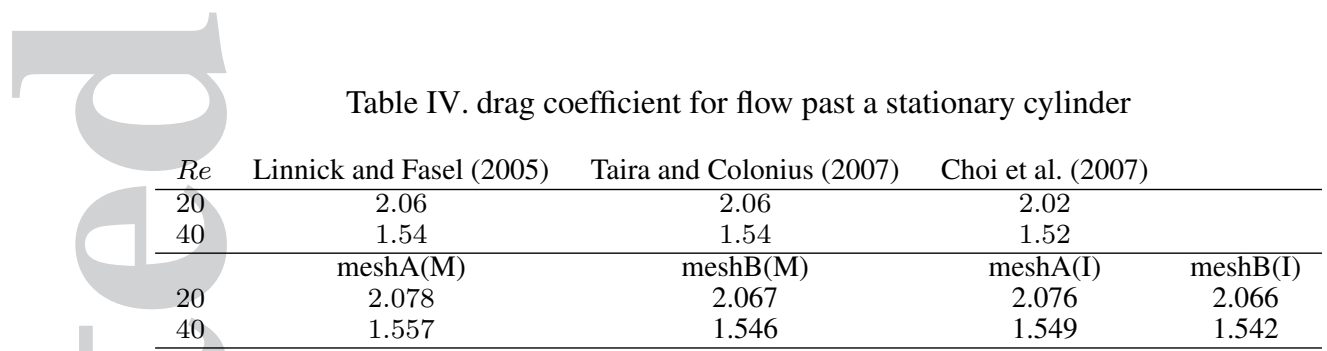

This article is protected by copyright. All rights reserved. 
Table V. drag and lift coefficients for flow past a stationary cylinder

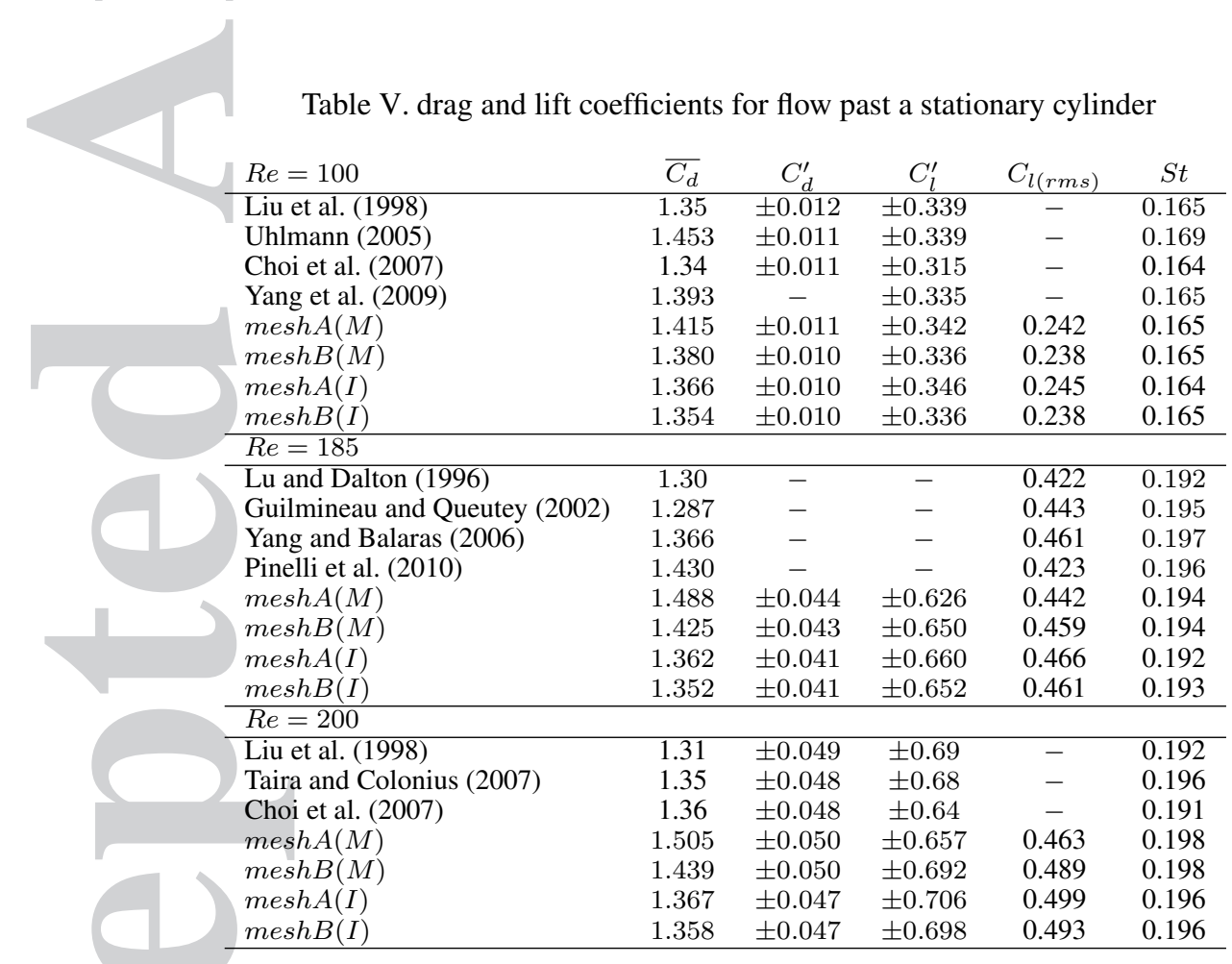

This article is protected by copyright. All rights reserved. 
Table VI. drag and lift coefficient for flow past an oscillatory cylinder

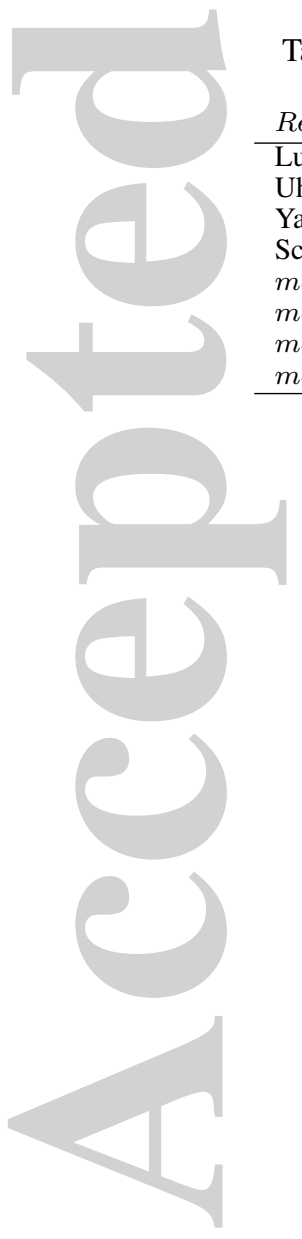

\begin{tabular}{|c|c|c|c|c|c|}
\hline$R e=185$ & $\overline{C_{d}}$ & $C_{d}^{\prime}$ & $C_{l}^{\prime}$ & $C_{l(r m s)}$ & St \\
\hline Lu and Dalton (1996) & 1.25 & - & - & 0.18 & - \\
\hline UhImann (2005) & 1.354 & 0.065 & - & 0.166 & - \\
\hline Yang et al. (2009) & 1.273 & - & - & 0.083 & - \\
\hline Schneiders et al. 2013) & 1.279 & - & - & $0.082 \sim 0.086$ & - \\
\hline $\operatorname{mesh} A(M)$ & 1.394 & 0.071 & 0.174 & 0.121 & 0.155 \\
\hline $\operatorname{mesh} B(M)$ & 1.328 & 0.066 & 0.151 & 0.104 & 0.156 \\
\hline $\operatorname{mesh} A(I)$ & 1.273 & 0.062 & 0.124 & 0.083 & 0.156 \\
\hline $\operatorname{mesh} B(I)$ & 1.263 & 0.061 & 0.119 & 0.082 & 0.156 \\
\hline
\end{tabular}

This article is protected by copyright. All rights reserved. 


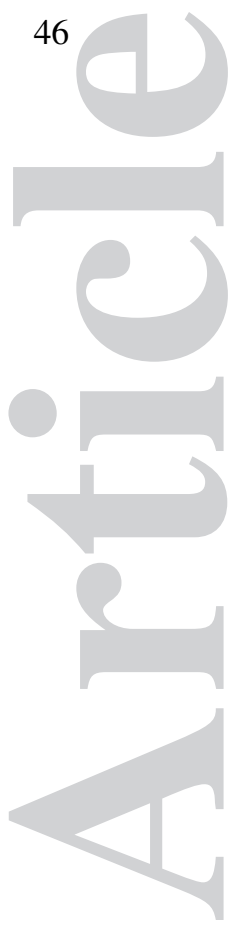

W. YI, D. CORBETT AND X.-F. YUAN

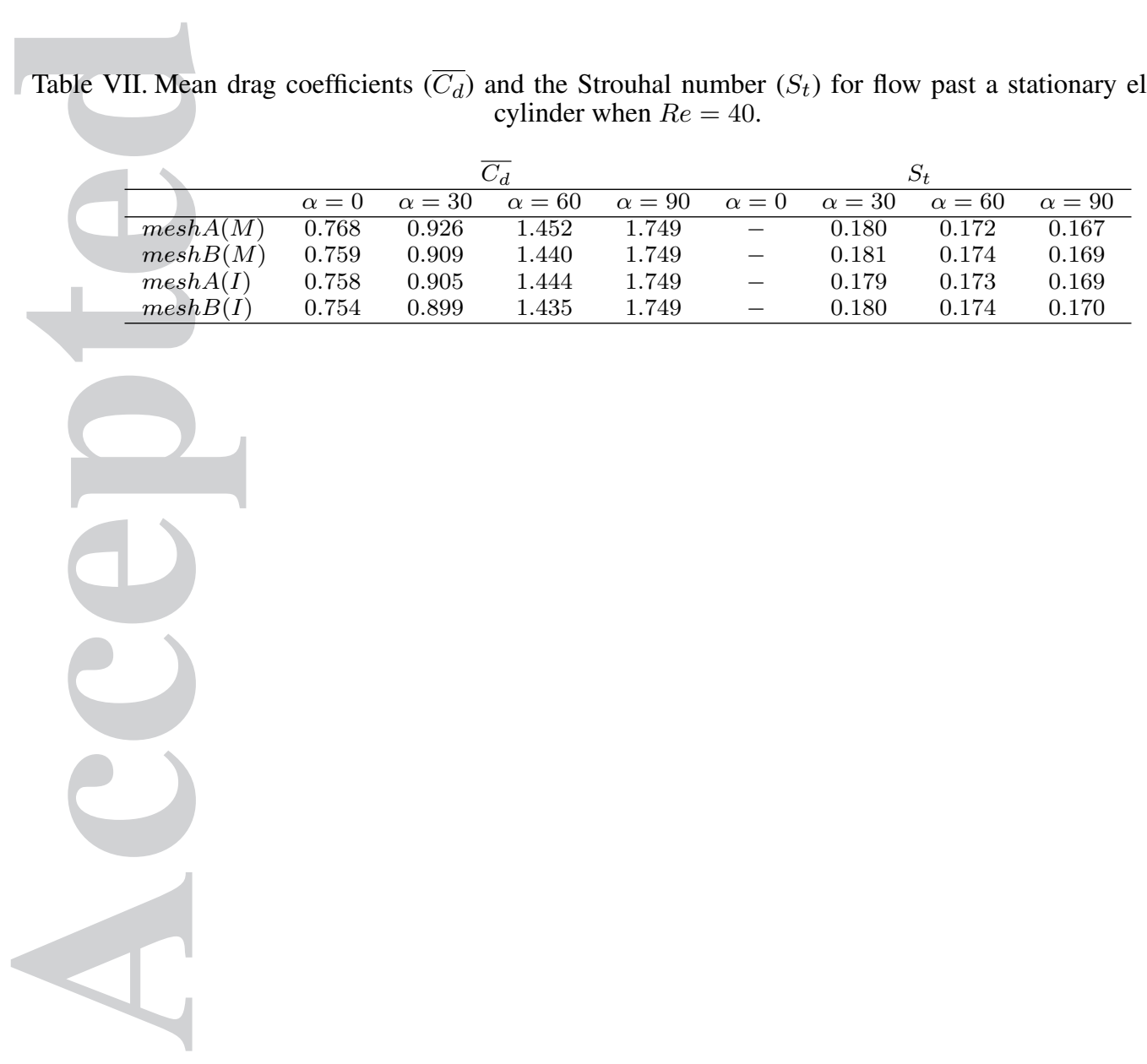

This article is protected by copyright. All rights reserved. 


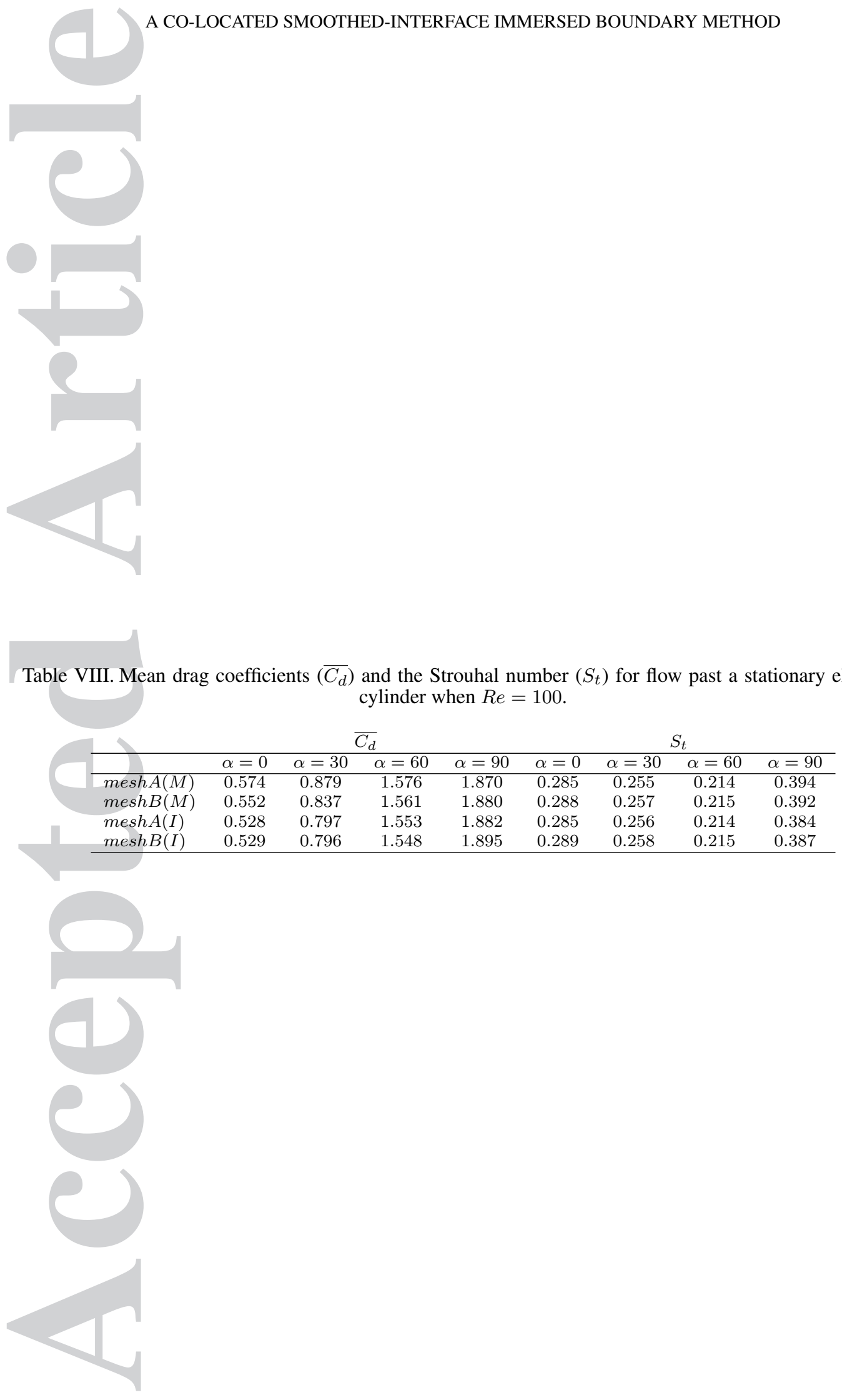

This article is protected by copyright. All rights reserved. 


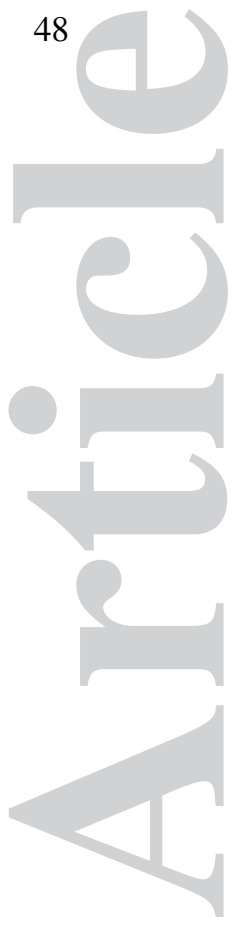

W. YI, D. CORBETT AND X.-F. YUAN

Table IX. Physical properties and geometric settings for disks

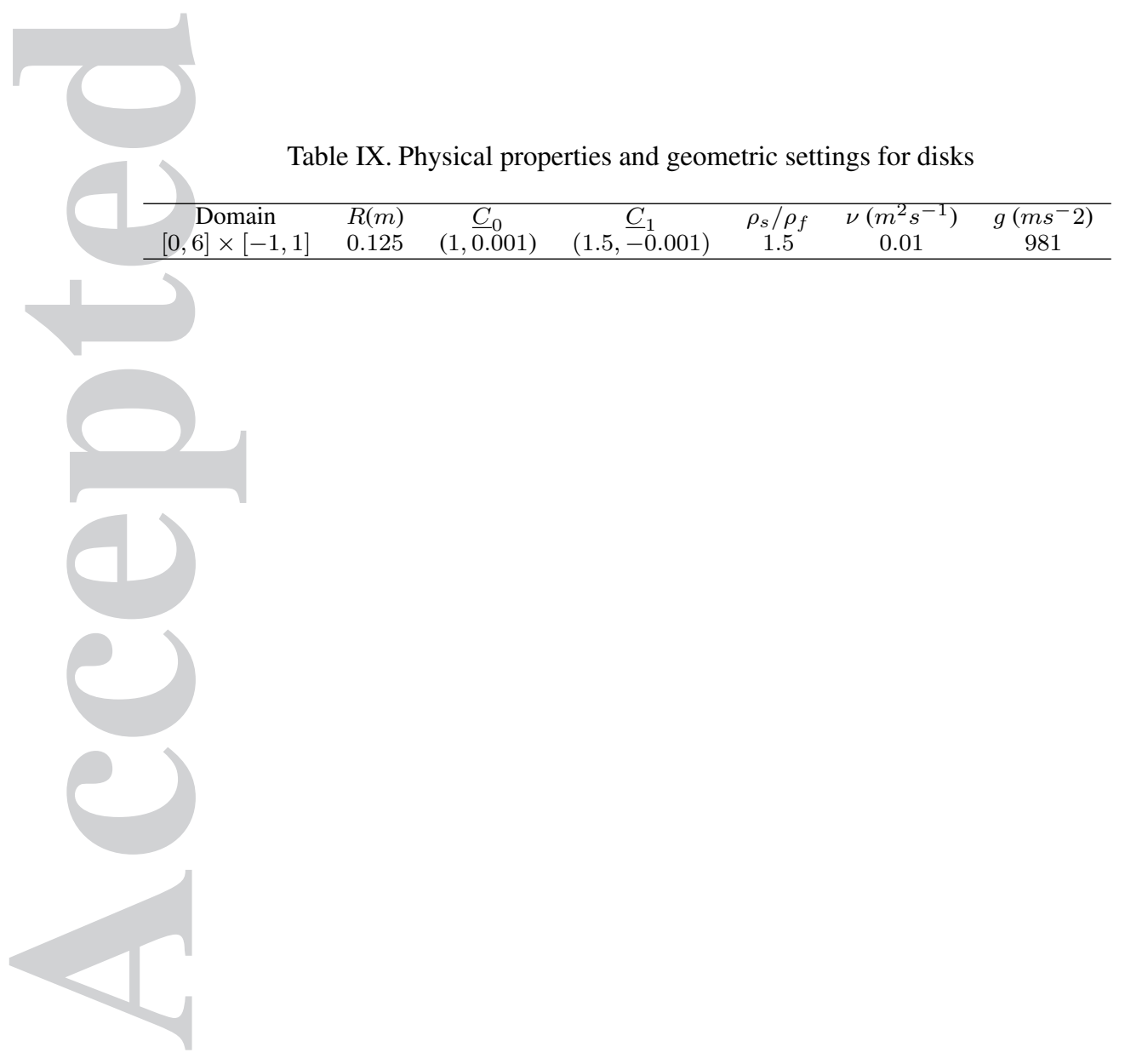

This article is protected by copyright. All rights reserved. 